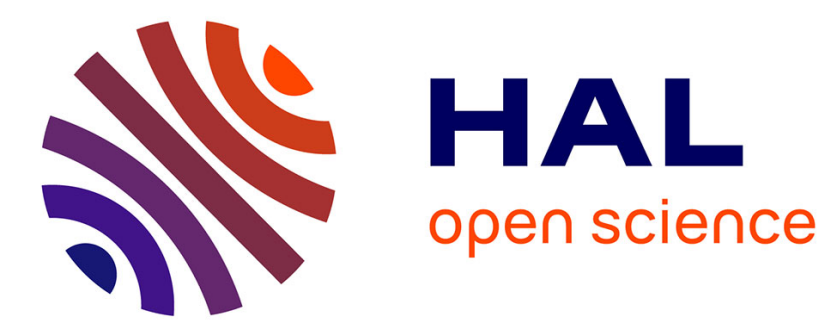

\title{
Large-eddy lattice-Boltzmann modeling of transonic flows
}

\author{
T. Coratger, G. Farag, S. Zhao, Pierre Boivin, P. Sagaut
}

\section{To cite this version:}

T. Coratger, G. Farag, S. Zhao, Pierre Boivin, P. Sagaut. Large-eddy lattice-Boltzmann modeling of transonic flows. Physics of Fluids, 2021, 33 (11), pp.115112. 10.1063/5.0064944 . hal-03424286

\section{HAL Id: hal-03424286 \\ https://hal.science/hal-03424286}

Submitted on 10 Nov 2021

HAL is a multi-disciplinary open access archive for the deposit and dissemination of scientific research documents, whether they are published or not. The documents may come from teaching and research institutions in France or abroad, or from public or private research centers.
L'archive ouverte pluridisciplinaire HAL, est destinée au dépôt et à la diffusion de documents scientifiques de niveau recherche, publiés ou non, émanant des établissements d'enseignement et de recherche français ou étrangers, des laboratoires publics ou privés. 


\title{
Large-eddy Lattice-Boltzmann modelling of transonic flows
}

\author{
T. Coratger, ${ }^{1}$ G. Farag, ${ }^{1}$ S. Zhao (赵峲), ${ }^{1}$ P. Boivin, ${ }^{1, \text { a) }}$ and P. Sagaut ${ }^{1}$ \\ Aix Marseille Univ, CNRS, Centrale Marseille, M2P2, Marseille,
} France

(Dated: November 5, 2021)

A D3Q19 Hybrid Recursive Regularized Pressure based Lattice Boltzmann Method (HRR-P LBM) is assessed for the simulation of complex transonic flows. Mass and momentum conservation equations are resolved through a classical LBM solver coupled with a finite volume resolution of entropy equation for a complete compressible solver preserving stability, accuracy and computational costs. An efficient treatment for wall and open boundaries is coupled with a grid refinement technique and extended to the HRR-P LBM in the scope of compressible aerodynamics. A Vreman subgrid turbulence model and an improved coupling of immersed boundary method with turbulence wall model on Cartesian grid accounts for unresolved scales by LargeEddy Simulation (LES). The validity of the present method for transonic applications is investigated through various test cases with increasing complexity starting from an inviscid flow over a $10 \%$ bump and ending with a turbulent flow over a ONERA M6 three-dimensional wing.

${ }^{a)}$ Electronic mail: pierre.boivin@univ-amu.fr 


\section{INTRODUCTION}

Lattice Boltzmann Methods (LBM) are an alternative powerful tool for the simulation of fluid dynamics ${ }^{1}$. Due to its attractive computational cost, its capacities for massively parallel computing and the ease to deal with complex geometries using multi-level Cartesian grids, these methods have shown a growing interest both in the academic and industrial spheres in the past decades 2 [4].

The development of LBM for compressible flows is still an open issue. Most existing methods on standard lattices (D1Q3, D2Q9 and D3Q19 or D3Q27) are restricted to isothermal or weakly compressible flows ${ }^{3}$, and thus are not applicable to high speed and high Reynolds flows, such as those found in most aerospace engineering related applications. To obtain a fully compressible solver, the well-known Mach error must be corrected ${ }^{5}$, coupled with a proper way to resolve energy.

In extending LB methods to compressible flows, the main difficulty is to account for energy conservation ${ }^{6}$. Two options are then available: (i) increase the number of discrete velocities as to accurately recover the moment of the probability function corresponding to energy, and (ii) solve the energy equation in a coupled manner. Approach (i) includes, e.g. multi-speed approaches ${ }^{7}$, but the number of discrete velocities (sometimes as high as $\left.7^{3}=343\right)$ seriously thwarts computational performances as compared with nearest-neighbor lattices, consisting of only 15 to 27 discrete velocities. In the second approach (ii), energy conservation is tackled separately, either as a second distribution function ${ }^{8}$ (in so-called double-distribution function methods), or simply as an additional scalar equation, solved using finite volumes ${ }^{\sqrt{59}}$ (often referred to as hybrid methods).

The hybrid formalism has recently attracted a lot of attention, because (i) it allows to account for any number of equations straightforwardly and without penalizing memory usage (e.g. for reactive flow applications $\frac{10}{14}$, pollutant dispersion $\frac{15}{17} \ldots$...), (ii) physical models implemented in classical Navier-Stokes solvers can be effortlessly transferred in the LB solver (since only mass and momentum equations differ), and (iii) they present excellent accuracy for the transport of elementary Kovasznay modes ${ }^{9[18 \mid 19}$.

Nonetheless, there remains a large gap between the complexity of full-scale industrial applications tackled by isothermal LB methods 20121 , and the compressible tests successfully validated using LBM: Kovasznay mode convections ${ }^{9}$, thermal Couette flow 22 24, 2D 
NACA0012 $2^{25 \mid 27}$, flow over bump ${ }^{2829}$, shock/vortex interaction ${ }^{930}$, Taylor-Green Vortex ${ }^{14431}$

and inviscid flow over wing ${ }^{32133}$. Filling that gap, and showing that our hybrid approach ${ }^{9118119}$ is now application-ready is the objective of the present study.

To that end, we present, for our recent pressure-based compressible LB mode 1919: (i) an algorithm to deal with mesh transitions, derived from state-of-the-art approaches ${ }^{33}$; 35 ; (ii) a subgrid turbulent model and a compatible wall-model adapted from the literatur ${ }^{36}{ }^{38}$; ; and (iii) an efficient shock-sensor. Equipped with these new developments, we present the first - to the authors' knowledge - wall-law large eddy Lattice-Boltzmann simulation of a 3D transonic flow.

The paper is organized as follows. Section [I] describes the key elements of the Hybrid Recursive Regularized Pressure based (HRR-P) model starting from the base of the LBM algorithm to the treatment of energy equation. Section [III investigates the LES turbulence model including Vreman subgrid model and the wall law description. Section IV] introduces new numerical ingredients for the pressure-based solver to handle complex geometries. Finally, numerical results obtained with this method are displayed in Section $\nabla$, followed by concluding remarks in Section VI

\section{LATTICE-BOLTZMANN MODEL FOR COMPRESSIBLE FLOWS}

\section{A. Macroscopic governing equations}

Before presenting the Lattice-Boltzmann model, let us introduce the corresponding macroscopic equations as well as necessary notations.

The motion of a fluid is described by the conservation laws of mass, momentum and energy.

$$
\begin{gathered}
\frac{\partial \rho}{\partial t}+\frac{\partial \rho u_{\beta}}{\partial x_{\beta}}=0, \\
\frac{\partial \rho u_{\alpha}}{\partial t}+\frac{\partial \rho u_{\alpha} u_{\beta}+\delta_{\alpha \beta} p-\Pi_{\alpha \beta}}{\partial x_{\beta}}=0, \\
\frac{\partial \rho E}{\partial t}+\frac{\partial \rho H u_{\alpha}}{\partial x_{\alpha}}=\frac{\partial \Pi_{\alpha \beta} u_{\alpha}}{\partial x_{\beta}}-\frac{\partial q_{\beta}}{\partial x_{\beta}},
\end{gathered}
$$

where $u_{\alpha}$ is the velocity vector, $\rho$ the volumic mass, $p$ the thermodynamic pressure, $E=$ $e+1 / 2 u_{\alpha} u_{\alpha}$ the total energy, and $H=E+p / \rho$ the total enthalpy. The internal energy $e$ is 
defined by $e=C_{v} T$, assuming constant volume heat capacity $C_{v}$. The viscous stress tensor $\Pi_{\alpha \beta}$ is

$$
\Pi_{\alpha \beta}=\mu\left(\frac{\partial u_{\alpha}}{\partial x_{\beta}}+\frac{\partial u_{\beta}}{\partial x_{\alpha}}-\delta_{\alpha \beta} \frac{2}{3} \frac{\partial u_{\gamma}}{\partial x_{\gamma}}\right),
$$

and the heat flux $q_{\beta}$ reads

$$
q_{\beta}=-\lambda \frac{\partial T}{\partial x_{\beta}},
$$

with $\mu$ the dynamic viscosity and $\lambda$ the heat conductivity. To close the above system, we consider an ideal equation of state

$$
p=\rho r T
$$

with $r=R / W$ the specific gas constant, $R$ is the universal gas constant, $W$ is the molecular weight. According to dry air properties, $r=2.9 \times 10^{2} \mathrm{~J} \mathrm{~kg}^{-1} \mathrm{~K}^{-1}$ will be considered in the following work. Assuming a calorically perfect gas (constant specific heat $C_{p}$ and $C_{v}$, for constant pressure and volume process respectively), the entropy can be defined as:

$$
s=C_{v} \ln \frac{p}{\rho^{\gamma}}
$$

where $\gamma=C_{p} / C_{v}$ is the adiabatic exponent.

\section{B. Lattice Boltzmann Method for mass and momentum conservation}

LB methods are based on the kinetic theory of gases at a mesoscopic scale, introducing a statistical representation of the fluid. The discrete description is done through the so-called particle distribution function $f_{i}\left(\mathbf{x}, \mathbf{c}_{\mathbf{i}}, t\right)$, function of the position $\mathbf{x}$, the particle velocity $\mathbf{c}_{\mathbf{i}}$ (discretized as a finite dimension velocity space) and the time $t$. In the absence of external forces, the Lattice Boltzmann Equation (LBE) can be derived from the Boltzmann equation in the velocity space as

$$
\frac{\partial f_{i}}{\partial t}+c_{i, \alpha} \frac{\partial f_{i}}{\partial x_{\alpha}}=\Omega_{i}
$$

$\Omega_{i}$ denotes the collision term. With the hypothesis of a single relaxation time collision operator, the so-called Bhatnagar-Gross-Krook (BGK) ${ }^{39}$ operator can be introduced:

$$
\Omega_{i}=-\frac{1}{\tau}\left(f_{i}-f_{i}^{\mathrm{eq}, 19 r}\right)
$$


where $\tau$ is the relaxation time toward equilibrium associated to the dynamic viscosity $\stackrel{40}{\text { : }}$

$$
\tau=\frac{\mu}{\rho c_{s}^{2}}
$$

Let us additionally introduce a dimensionless temperature $\theta$ as

$$
\theta=\frac{T}{T_{0}},
$$

Where $T_{0}$ is a reference temperature, corresponding to a reference velocity $c_{0}$.

$$
c_{0}=\sqrt{r T_{0}}=c_{s} \frac{\Delta x}{\Delta t}
$$

The collision step brings the distribution $f_{i}$ back to a modified pressure-based ${ }^{9}$ third order equilibrium distribution $f_{i}^{\text {eq, } 19 r}$

$$
\begin{array}{r}
f_{i}^{\text {eq, } 19 r}=\omega\left\{\rho \theta+\frac{\mathcal{H}_{i \alpha}^{(1)}}{c_{s}^{2}} \rho u_{\alpha}+\frac{\mathcal{H}_{i \alpha \beta}^{(2)}}{2 c_{s}^{4}} \rho u_{\alpha} u_{\beta}\right. \\
+\frac{1}{6 c_{s}^{6}}\left[\quad+3\left(\mathcal{H}_{i, x x y}^{(3)}+\mathcal{H}_{i, y z z}^{(3)}\right)\left(\rho u_{x} u_{x} u_{y}+\rho u_{y} u_{z} u_{z}\right)\right. \\
+\left(\mathcal{H}_{i, x x y}^{(3)}-\mathcal{H}_{i, y z z}^{(3)}\right)\left(\rho u_{x} u_{x} u_{y}-\rho u_{y} u_{z} u_{z}\right) \\
+3\left(\mathcal{H}_{i, x z z}^{(3)}+\mathcal{H}_{i, x y y}^{(3)}\right)\left(\rho u_{x} u_{z} u_{z}+\rho u_{x} u_{y} u_{y}\right) \\
+\left(\mathcal{H}_{i, x z z}^{(3)}-\mathcal{H}_{i, x y y}^{(3)}\right)\left(\rho u_{x} u_{z} u_{z}-\rho u_{x} u_{y} u_{y}\right) \\
+3\left(\mathcal{H}_{i, y y z}^{(3)}+\mathcal{H}_{i, x x z}^{(3)}\right)\left(\rho u_{y} u_{y} u_{z}+\rho u_{x} u_{x} u_{z}\right) \\
\left.\left.+\left(\mathcal{H}_{i, y y z}^{(3)}-\mathcal{H}_{i, x x z}^{(3)}\right)\left(\rho u_{y} u_{y} u_{z}-\rho u_{x} u_{x} u_{z}\right)\right]\right\} .
\end{array}
$$

The equilibrium function $f_{i}^{\text {eq, } 19 r}$ is expressed in the $D 3 Q 19$ rotational symmetry basis of Gauss-Hermite polynomials to reduce the defect of the third order moment $\frac{36}{3}$. In the pressure-based framework, the moments of the equilibrium distribution function Eq. (13) (denoted as $f_{i}^{e q}$ for the sake of simplicity) on the nearest neighbor lattices type are:

$$
\begin{gathered}
\sum_{i} f_{i}^{e q}=\rho \theta \\
\sum_{i} c_{i, \alpha} f_{i}^{e q}=\rho u_{\alpha}, \\
\sum_{i} c_{i, \alpha} c_{i, \beta} f_{i}^{e q}=\rho u_{\alpha} u_{\beta}+\rho \theta c_{s}^{2} \delta_{\alpha \beta} .
\end{gathered}
$$

$\mathcal{H}_{i}$ are discrete Hermite polynomials in the velocity space, defined by:

$$
\begin{gathered}
\mathcal{H}_{i}^{(0)}=1, \\
\mathcal{H}_{i \alpha}^{(1)}=c_{i \alpha}, \\
\mathcal{H}_{i \alpha \beta}^{(2)}=c_{i \alpha} c_{i \beta}-c_{s}^{2} \delta_{\alpha \beta}, \\
\mathcal{H}_{i \alpha \beta \gamma}^{(3)}=c_{i \alpha} c_{i \beta} c_{i \gamma}-c_{s}^{2}\left[c_{i \alpha} \delta_{\beta \gamma}+c_{i \beta} \delta_{\gamma \alpha}+c_{i \gamma} \delta_{\alpha \beta}\right],
\end{gathered}
$$


The Lattice Boltzmann equation is then discretized in space and time using a trapezoidal $\operatorname{method~}^{3}$

$$
f_{i}\left(x_{\alpha}+c_{i \alpha} \Delta t, t+\Delta t\right)-f_{i}\left(x_{\alpha}, t\right)=\frac{\Delta t}{2}\left[\Omega_{f_{i}}\left(x_{\alpha}+c_{i \alpha} \Delta t, t+\Delta t\right)+\Omega_{f_{i}}\left(x_{\alpha}, t\right)\right]
$$

Eq. (21) is implicit since the collision term at $t+\Delta t$ depends on the distribution function $f_{i}$ at the same time step. To remove the implicit treatment of this equation and to ensure a second-order accuracy in time, a new distribution function is introduced, namely:

$$
\bar{f}_{i}=f_{i}-\frac{\Delta t}{2 \tau}\left(f_{i}^{\mathrm{eq}}-f_{i}\right)
$$

associated to a new relaxation time:

$$
\bar{\tau}=\tau+\frac{\Delta t}{2}
$$

The collision algorithm leading to the post-collide population at time step $t+\Delta t$ can be summarized as:

$$
\bar{f}_{i}^{\mathrm{col}}\left(t+\Delta t, x_{\alpha}\right)=f_{i}^{\mathrm{eq}}\left(t+\Delta t, x_{\alpha}\right)+\left(1-\frac{\Delta t}{\bar{\tau}}\right) \bar{f}_{i}^{\mathrm{neq}}\left(t+\Delta t, x_{\alpha}\right)+\frac{\Delta t}{2} F_{i}^{E}\left(t+\Delta t, x_{\alpha}\right)
$$

in which the forcing term $F_{i}^{E}$ has been introduced, as described in Appendix A, to recover an accurate non-equilibrium tensor and to correct the defect of isotropy of the lattice $\mathrm{e}^{918141}$.

A hybrid recursive regularized collision kerne ${ }^{36}(\mathrm{HRR})$ is used to recover a correct viscous stress tensor.

Regularization procedures of the distribution function $f_{i}$ were proposed to improve the numerical stability of BGK collision models in pioneering works ${ }^{\sqrt{36142143}}$ by filtering out spurious non-hydrodynamic modes. Hence, a regularized distribution function is introduced prior to the collision step through recomputing the non-equilibrium parts. This involves a truncation up to second order, projected on the Hermite polynomial basis. The non-equilibrium regularized population $\bar{f}_{i}^{\text {neq }}$ is evaluated as

$$
\bar{f}_{i}^{\text {neq }} \equiv \omega_{i}\left[\frac{\mathcal{H}_{i, \alpha \beta}^{(2)}}{2 c_{s}^{4}} \tilde{a}_{\alpha \beta}^{(2), \text { neq }}+\frac{\mathcal{H}_{i, \gamma}^{(3 r)}}{6 c_{s}^{6}} a_{\gamma}^{(3 r), \text { neq }}\right],
$$

with the third order off-equilibrium $a_{\gamma}^{(3 r) \text {,neq }}$ recursively derived as detailed in Appendix B.

In the hybrid recursive regularized collision kerne ${ }^{[36}, \tilde{a}_{\alpha \beta}^{(2) \text {,neq }}$ is decomposed into two contributions. On one hand, $\bar{a}_{\alpha \beta}^{(2), \text { neq,PR }}$ is the direct projection of $\bar{f}_{i}^{\text {neq }}$ as:

$$
\bar{a}_{\alpha \beta}^{(2) \text {,neq,PR }}=\sum_{i=1}^{19}\left[\left(\bar{f}_{i}-f_{i}^{\text {eq }}+\frac{\Delta t}{2} F_{i}^{E}\right) \mathcal{H}_{i \alpha \beta}^{(2)}\right]
$$


On the other hand, $\bar{a}_{\alpha \beta}^{(2), \text { neq,FD }}$ is the approximation of the Navier-Stokes viscous stress tensor resolved by second order finite differences in the core of the fluid and a first order decentered procedure for the boundary conditions.

$$
\bar{a}_{\alpha \beta}^{(2), \text { neq,FD }}=-\rho c_{s}^{2} \bar{\tau}\left(\frac{\partial u_{\alpha}}{\partial x_{\beta}}+\frac{\partial u_{\beta}}{\partial x_{\alpha}}-\delta_{\alpha \beta} \frac{2}{3} \frac{\partial u_{\gamma}}{\partial x_{\gamma}}\right),
$$

Improved stability ${ }^{40}$ is achieved by numerically enforcing a traceless stress tensor, which

allows to filter an additional non-hydrodynamic mode 44 . Finally, the stress tensor $\tilde{a}_{\alpha \beta}^{(2), \text { neq }}$ in the non-equilibrium part 25 reads 36 :

$$
\tilde{a}_{\alpha \beta}^{(2), \text { neq }} \equiv \sigma\left[\bar{a}_{\alpha \beta}^{(2), \text { neq,PR }}-\frac{\delta_{\alpha \beta}}{3} \bar{a}_{\gamma \gamma}^{(2), \text { neq,PR }}\right]+(1-\sigma) \bar{a}_{\alpha \beta}^{(2), \text { neq,FD }},
$$

with $\sigma \in[0,1]$ a free parameter. Then, knowing the collide population at time step $t$, an intermediate population $\bar{f}_{i}$ is obtained by a streaming step from neighbors

$$
\bar{f}_{i}(t+\Delta t, \boldsymbol{x})=\bar{f}_{i}^{\mathrm{col}}\left(t, \boldsymbol{x}-\boldsymbol{c}_{\boldsymbol{i}} \Delta t\right) .
$$

This post-streaming population acts as a base for the whole next time step calculations and the well-known stream and collide algorithm is recovered.

\section{A finite difference method for entropy equation}

To couple the energy conservation with the LBM solver, an entropy equation under nonconservative format is solved ${ }^{18}$ using second order finite difference:

$$
\underbrace{\frac{\partial s}{\partial t}+u_{\beta} \frac{\partial s}{\partial x_{\beta}}}_{\text {MUSCL-Hancock }}=\underbrace{\frac{1}{\rho T}\left[\Pi_{\alpha \beta} \frac{\partial u_{\alpha}}{\partial x_{\beta}}-\frac{\partial q_{\beta}}{\partial x_{\beta}}\right]}_{\text {Finite differences - Second order }}
$$

The temporal derivative and the convective flux in Eq. (30) are discretized using a MUSCLHancock method (see Appendix C for details). This scheme is non-local and requires a fivepoints stencil per direction. Near boundary conditions, this stencil is not always available, and a mix ${ }^{9}$ of second order centered flux (to properly include the boundary condition) with upwinding (for numerical stability) is used. Thermal conduction and viscous heat are computed through a simple second order centered finite difference scheme, involving a first order decentered procedure for near-boundaries nodes.

Note that we recently proposed a numerically conservative scheme ${ }^{\sqrt{45}}$ to solve directly a total energy equation, but applying it to configurations as complex as presented hereafter is still under investigation. 


\section{FILTERED EQUATIONS: TURBULENCE AND WALL LAW MODEL}

\section{A. Vreman subgrid turbulence model}

In the context of Large Eddy Simulations (LES) $)^{46}$, the filtered macroscopic equations (1.3) are

$$
\begin{aligned}
\frac{\partial \bar{\rho}}{\partial t}+\frac{\partial \bar{\rho} \tilde{u}_{\alpha}}{\partial x_{\alpha}} & =0 \\
\frac{\partial \bar{\rho} \tilde{u}_{\alpha}}{\partial t}+\frac{\partial \bar{\rho} \tilde{u}_{\alpha} \tilde{u}_{\beta}}{\partial x_{\beta}} & =-\frac{\partial \bar{p}}{\partial x_{\alpha}}+\frac{\partial}{\partial x_{\beta}}\left(\tilde{\Pi}_{\alpha \beta}-R_{\alpha \beta}\right), \\
\frac{\partial \bar{s}}{\partial t}+\bar{u}_{\alpha} \frac{\partial \bar{s}}{\partial x_{\alpha}} & =\frac{1}{\bar{\rho} \bar{T}} \frac{\partial}{\partial x_{\alpha}}\left(\lambda \frac{\partial \bar{T}}{\partial x_{\alpha}}-Q_{\alpha}^{s g s}\right)+\frac{1}{\bar{\rho} \bar{T}} \bar{\Pi}_{\alpha \beta} \frac{\partial \bar{u}_{\alpha}}{\partial x_{\beta}},
\end{aligned}
$$

where $\overline{()}$ are the grid-filtered quantities and $\tilde{()}$ indicates Favre filter $\tilde{u}_{\alpha} \equiv \overline{\rho u_{\alpha}} / \bar{\rho}$. $R_{\alpha \beta}$ is the unknown SGS Reynolds stress tensor $R_{\alpha \beta}=\bar{\rho}\left(\widetilde{u_{\alpha} u_{\beta}}-\tilde{u}_{\alpha} \tilde{u}_{\beta}\right)$ and $Q_{\alpha}^{s g s}$ is the sum of

all subgrid contributions coming from the non-linearities in the entropy equation. Here, the unresolved heat flux will therefore follow from the eddy viscosity model. For the Vreman model ${ }^{47}$, the most important subgrid-term is the stress tensor $R_{\alpha \beta}$ which satisfies:

$$
R_{\alpha \beta}-\frac{1}{3} R_{\gamma \gamma} \delta_{\alpha \beta}=-2 \mu_{t}\left(\tilde{S}_{\alpha \beta}-\frac{1}{3} \tilde{S}_{\gamma \gamma} \delta_{\alpha \beta}\right),
$$

with $\tilde{S}_{\alpha \beta}=\frac{1}{2}\left(\frac{\partial \tilde{u}_{\alpha}}{\partial x_{\beta}}+\frac{\partial \tilde{u}_{\beta}}{\partial x_{\alpha}}\right), \delta_{\alpha \beta}$ is the usual Kronecker symbol and $\mu_{t}$ the turbulent viscosity. Vreman ${ }^{48}$ proposed a subgrid model where

$$
\mu_{t}=c \sqrt{\frac{B_{\beta}}{\alpha_{i j} \alpha_{i j}}}
$$

with

$$
\begin{aligned}
\alpha_{i j} & =\frac{\partial \tilde{u}_{j}}{\partial x_{i}}, \\
\beta_{i j} & =\Delta_{m}^{2} \alpha_{m i} \alpha_{m j}, \\
B_{\beta} & =\beta_{x x} \beta_{y y}-\beta_{x y}^{2}+\beta_{x x} \beta_{z z}-\beta_{x z}^{2}+\beta_{y y} \beta_{z z}-\beta_{y z}^{2} .
\end{aligned}
$$

In Eq. (35), $c$ is related to the Smagorinsky constant $C_{s}=0.18$ such that $c=2.5 C_{s}^{2}$. This turbulent viscosity introduced in the momentum equation is coupled with a turbulent thermal conductivity to model nonlinearities in entropy equation:

$$
\begin{array}{r}
Q_{\alpha}^{s g s}=-\lambda_{s g s} \frac{\partial \bar{T}}{\partial x_{\alpha}}, \\
\lambda_{s g s}=\frac{\mu_{t} c_{p}}{\operatorname{Pr}_{t}},
\end{array}
$$

where the turbulent Prandtl number is set as $\operatorname{Pr}_{t}=0.9$, following Garnier et al. $\stackrel{49}{\text {. }}$ 


\section{B. An explicit wall model for HRR LBM solver}

For turbulent flows (at high Reynolds numbers), solving directly the flow conservation equations near the walls requires a huge number of points, a procedure which is not affordable for industrial applications. Hence, to reduce computational efforts, algebraic explicit wall models are proposed in the literature to cover the entire inner region of the turbulent boundary layer. In this study, logarithmic laws of the wall are used to calculate the friction velocity on boundary points, considering the cartesian grid model inherited from the LBM mesh. Non dimensional quantities:

$$
u^{+}=\frac{u}{u_{\tau}} \quad \text { or } \quad y^{+}=\frac{y u_{\tau}}{\nu},
$$

with $u_{\tau}$ the friction velocity, $y$ the distance to the wall and $\nu$ the molecular kinematic viscosity are expressed as a function of the local Reynolds number:

$$
\operatorname{Re}_{y}=\frac{u y}{\nu}=u^{+} y^{+},
$$

implying for the friction velocity:

$$
u_{\tau}=\frac{u}{u^{+}\left(\operatorname{Re}_{y}\right)} \quad \text { or } \quad u_{\tau}=\frac{y^{+}\left(\operatorname{Re}_{y}\right) \nu}{y} .
$$

The turbulent boundary layer can be divided into three parts: the viscous sublayer, the buffer layer and the inertial layer. In the viscous sublayer:

$$
u^{+}=y^{+}=\sqrt{\operatorname{Re}_{y}} .
$$

In the inertial layer, the velocity follows a logarithmic law:

$$
u^{+}=\frac{1}{\kappa} \log \left(E y^{+}\right) \quad \text { or } \quad y^{+}=\frac{1}{E} \exp \left\{\kappa u^{+}\right\},
$$

where $\kappa \approx 0.41$ and $E \approx 7.9$. Following a sixth order series expansion ${ }^{50}$ of the Lambert function $W(x)$, the following explicit version of the logarithmic profile on $y^{+}\left(\operatorname{Re}_{y}\right)$ in the inertial layer is obtained:

$$
y^{+}\left(\operatorname{Re}_{y}\right)=\frac{1}{E} \exp \left\{W\left(\kappa E \operatorname{Re}_{y}\right)\right\},
$$

Then, a unified expression for the entire layer can be given in the form:

$$
\begin{array}{r}
y^{+}\left(\operatorname{Re}_{y}\right)=\left(1-\tanh \frac{\operatorname{Re}_{y}}{s}\right)^{p}\left(\operatorname{Re}_{y}\right)^{1 / 2}+\left(\tanh \frac{\operatorname{Re}_{y}}{s}\right)^{p} \frac{1}{E} \exp \left\{W\left(\kappa E \operatorname{Re}_{y}\right)\right\} . \\
u^{+}\left(\operatorname{Re}_{y}\right)=\frac{\operatorname{Re}_{y}}{y^{+}\left(\operatorname{Re}_{y}\right)},
\end{array}
$$


with the constants $p=0.7894$ and $s=86.58$ calibrated to the Spalart-Allmaras turbulence model to minimize the error between the curve $y^{+}\left(\operatorname{Re}_{y}\right)$ and the target one, taking into account the three zones of the turbulent boundary layer. The above formulae are used for $\kappa E \operatorname{Re}_{y}>e$ with $e$ being the natural number, to ensure convergence of the expansion. Otherwise, Eq. (44) should be used. Then, $u_{\tau}$ can be deduced using Eq. (43). Following the Spalart-Allmaras turbulence model, $u^{+}$can be written using arithmetic 51 :

$$
\begin{array}{r}
u^{+}\left(y^{+}\right)=\bar{B}+c_{1} \log \left(\left(y^{+}+a_{1}\right)^{2}+b_{1}^{2}\right)-c_{2} \log \left(\left(y^{+}+a_{2}\right)^{2}+b_{2}^{2}\right) \\
-c_{3} \operatorname{atan} 2\left(y^{+}+a_{1}, b_{1}\right)-c_{4} \operatorname{atan} 2\left(y^{+}+a_{2}, b_{2}\right),
\end{array}
$$

where $\operatorname{atan} 2(x, y)$ returns the principal value of the arc tangent of $x / y$, expressed in radians. The values of the coefficients $\left(\bar{B}, a_{1}, a_{2}, b_{1}, b_{2}, c_{1}, c_{2}, c_{3}\right.$ and $\left.c_{4}\right)$ can be found in $\frac{51}{\text {. The }}$ definition of the tangential velocity follows:

$$
u=u^{+} u_{\tau}
$$

\section{NUMERICAL SETUP}

\section{A. An artificial viscosity method to capture shocks}

The aim of shock capturing techniques is to automatically reduce the Gibbs phenomenon 52 that creates oscillations near discontinuities. The methods commonly exploit the effect of dissipative mechanisms on shocks. The idea is to add artificial dissipation through numerical viscosity into the conservation equations to increase the thickness of the shock and to make it of the order of (or larger than) the mesh size. The first step is to detect shocks properly. For that, a pressure shock sensor based on a Jameson-Schmidt-Turkel ${ }^{53}$ (JST) scheme is used:

$$
\nu_{s c}=\frac{\left|p_{i-1}-2 p_{i}+p_{i+1}\right|}{\left|p_{i-1}+2 p_{i}+p_{i+1}\right|} .
$$

This result is turned into an artificial kinetic viscosity modelled by a free parameter $s_{c}$ chosen by the user. Hence, the effective relaxation time can be summed as:

$$
\bar{\tau}_{e}=\frac{\mu+\rho \nu_{s c} s_{c}}{\rho c_{s}^{2}}+\frac{\Delta t}{2} .
$$




\section{B. Immersed solid boundaries via cut cell approach}

The boundary conditions of the pressure-based distribution function are implemented by a finite difference reconstruction approach along with the hybrid regularization procedure ${ }^{42}$.

First, the macroscopic quantities $\rho, \mathbf{u}, \theta$ and $s$ are computed. A cut cell method is adopted to handle solid walls and open boundaries. Macroscopic quantities are calculated at two reference points with a Shepard's Inverse Distance Weighting (IDW) algorithm. Then, the boundary node is given by a second order Lagrangian interpolation to obtain a proper Dirichlet or Neumann condition ${ }^{26}$.

Next, the viscous stress tensor is computed using its Navier-Stokes format Eq. (4) and the velocity gradients are properly decentered with a first order algorithm. The non equilibrium part of the distribution function is finally recovered using Eq. (28) with $\sigma=0$.

\section{Open boundary conditions}

For far-field open boundaries and subsonic flows, characteristic boundary conditions are used. Local One-Dimensional Inviscid (LODI) ${ }^{54}$ are extended and evaluated within the hybrid thermal lattice Boltzmann framework. The time evolution of a closed system is governed by the state in the interior of the domain and by waves entering the domain through its boundaries. In a characteristic-based nonreflecting boundary condition, the time derivatives of physical quantities at the boundary are modified. The first goal of this filter is to minimize the spurious reflection of characteristic waves leaving the domain and the second one is to enforce the desired value of incoming waves. In the framework of NSCBC boundary conditions, the amplitude of outgoing waves can be calculated using interior points and previous time step. But, it is impossible to determine the exact values of the incoming waves. Approximations have to be done to express the unknown $L_{i}$ 's (incoming waves) as a function of the known $L_{i}$ 's (outgoing waves).

To implement the characteristic subsonic outflow boundary condition with a prescribed static pressure $p_{\infty}$ and to avoid spurious pressure drifts, a partially non-reflecting outflow is built using:

$$
L_{1}=K_{\mathrm{cbc}}\left(p-p_{\infty}\right),
$$

where $K_{\mathrm{cbc}}$ has to be changed as a function of a dimensional parameter depending on the 
test case ${ }^{54}$. Through numerical experiments over transonic test case, it was found that $K_{\mathrm{cbc}}=0.05$ was a stable and robust value for all the cases mentioned in Section $\mathrm{V}$. After this step, the reconstruction procedure is the same as mentioned before for solid boundaries.

\section{Absorbing sponge layer}

NSCBC boundary conditions are supplemented by absorbing sponge terms. The compressible Navier-Stokes equations are artificially modified using source terms as follows:

$$
\begin{aligned}
\frac{\partial \rho}{\partial t}+\frac{\partial\left(\rho u_{\alpha}\right)}{\partial x_{\alpha}} & =-\sigma_{s}(x)\left(\rho-\rho_{f}\right), \\
\frac{\partial\left(\rho u_{\alpha}\right)}{\partial t}+\frac{\partial}{\partial x_{\beta}}\left(\rho u_{\alpha} u_{\beta}+p \delta_{\alpha \beta}\right) & =\frac{\partial \prod_{\alpha \beta}}{\partial x_{\beta}}-\sigma_{s}(x)\left(\rho u_{\alpha}-\rho_{f} u_{\alpha, f}\right), \\
\frac{\partial s}{\partial t}+u_{\alpha} \frac{\partial s}{\partial x_{\alpha}} & =\frac{1}{\rho T} \frac{\partial}{\partial x_{\alpha}}\left(\lambda \frac{\partial T}{\partial x_{\alpha}}\right)+\frac{1}{\rho T} \prod_{\alpha \beta} \frac{\partial u_{\alpha}}{\partial x_{\beta}}-\sigma_{s}(x)\left(s-s_{f}\right),
\end{aligned}
$$

Where $\rho_{f}, u_{\alpha, f}$ and $s_{f}$ are the prescribed density, velocity and entropy at the reference state, respectively. The sponge terms on the right-hand side are activated near open boundaries in order to damp the fluctuations via a relaxation toward a prescribed reference state.

In the absorbing layers, the profile $\sigma_{s}(x)$ could not be a uniformly distributed parameter. This will induce a significant wave reflection from the interfaces between the wave propagation domain and the sponge domain. Several definitions of this parameter exist. In this study, we will use

$$
\sigma_{s}(x)=\sigma_{A} \frac{3125\left(x-x_{0}\right)^{4}\left(L_{s}-x\right)}{256\left(L_{s}-x_{0}\right)^{5}},
$$

where $\sigma_{A}, x_{0}$ and $L_{s}$ are respectively the sponge strength factor to tune, starting position and thickness of the absorbing sponge layer, respectively.

\section{E. 3D Grid refinement for compressible HRR-LBM}

The grid refinement method for three-dimensional grid is based on multidomain decomposition. The space is discretized with a hierarchy of uniform embedded meshes, including a grid step ratio of 2 between two successive refinement areas. In this section, only the principal formalism and major equations are presented. Detailed description and explanatory 
schemes can be found in 33 . The mesh size and time step are defined as:

$$
\begin{aligned}
\Delta x_{f} & =\frac{1}{2} \Delta x_{c}=\frac{1}{2^{(L+1)}} \Delta x_{0}, \\
\Delta t_{f}=\frac{1}{2} \Delta t_{c} & =\frac{1}{2^{(L+1)}} \Delta t_{0},
\end{aligned}
$$

With $\Delta x_{0}$ and $\Delta t_{0}$ the mesh size and the time step at the coarsest level $L=0$. Subscripts $\mathrm{f}$ and $\mathrm{c}$ denote respectively the fine and coarse grid. With this approach, the stream and collide algorithm cannot be applied as it for a transition node at the interface between a fine and a coarse mesh. The detailed communication algorithm between the different levels is given in ${ }^{33}$. An interpolation step is needed at the transition interface. The time interpolation is due to the synchronisation of informations at odd and even time steps. During even time steps, informations on coarse and fine points are synchronous while they must be interpolated from coarse to fine grid at odd time steps:

$$
\phi_{f}^{(f)}=\phi_{f}^{\left(f-\Delta t_{f}\right)}+\frac{\phi_{c}^{\left(f+\Delta t_{f}\right)}-\phi_{f}^{\left(f-2 \Delta t_{f}\right)}}{3},
$$

where $\phi_{c}^{\left(f+\Delta t_{f}\right)}$ is obtained in advance on the coarse mesh. For transition points on the fine side which are not in the overlapped region, namely edge and face interfaces, spatial interpolations are performed, respectively in one and two dimensions. An automatic switch to asymmetric scheme is adopted when the number of neighbors if not fulfilled ${ }^{33}$. To better illustrate these words, examples of these cubic interpolations are provided (with $i, j$ and $k$ respectively the index in $x, y$ and $z$ directions):

- One-dimensional edge interpolation

$$
\phi(i, j, k)=-\frac{1}{16} \phi(i-3, j, k)+\frac{9}{16} \phi(i-1, j, k)+\frac{9}{16} \phi(i+1, j, k)-\frac{1}{16} \phi(i+3, j, k),
$$

- Two-dimensional face interpolation on $x-y$ plane

$$
\begin{aligned}
\phi(i, j, k)=\frac{1}{2}\left[-\frac{1}{16} \phi(i-3, j, k)+\frac{9}{16} \phi(i-1, j, k)\right. \\
\left.+\frac{9}{16} \phi(i+1, j, k)-\frac{1}{16} \phi(i+3, j, k)\right] \\
+\frac{1}{2}\left[-\frac{1}{16} \phi(i-3, j, k)+\frac{9}{16} \phi(i-1, j, k)\right. \\
\left.+\frac{9}{16} \phi(i+1, j, k)-\frac{1}{16} \phi(i+3, j, k)\right] .
\end{aligned}
$$




\section{F. Coupling of wall model with immersed boundaries on Cartesian grids}

Spurious oscillations are frequently observed near surfaces where wall model is applied in the context of turbulent flows, especially on Cartesian grids 38 . The problem is well known and comes from the stair-step grid boundaries, inducing irregularities on the wall distances. To reduce these oscillations, several modifications are picked from ${ }^{38}$.

The first ingredient is to work on $u_{\tau}$ interpolations. As it is constant on the wall normal direction, interpolation can be performed only in the wall parallel direction. Usually, macroscopic quantities are interpolated and $u_{\tau}$ is deduced from the law of the wall. Direct interpolation of $u_{\tau}$ avoids the use of quantities following a rapid change. The tangential velocity at each neighbour points is calculated through:

$$
\mathbf{u}_{t}=\mathbf{u}-(\mathbf{u} . \mathbf{n}) \mathbf{n},
$$

with the parallel distance obtained from:

$$
d_{t, k}^{2}=\left\|\mathbf{x}_{B}-\mathbf{x}_{k}\right\|^{2}-\left\|\left(\mathbf{x}_{B}-\mathbf{x}_{k}\right) \cdot \mathbf{n}\right\|^{2} .
$$

The inverse is used as a weight to interpolate $u_{\tau}$, inverting the wall function. As in $\frac{56}{\text {, }}$ the interpolation support domain includes two layers of points, starting from the boundary node, in the D3Q19 lattice. Moreover, as the interpolation takes into account only parallel directions, the order of interpolation is reduced by one.

We observed through numerical experiments that evaluation of the velocity gradients near the wall is a main source of spurious oscillations. Classicaly, gradients are decentered near boundaries to achieve a first order scheme. This treatment seems not to be adequate for turbulent boundary layers where steep gradients are present near the wall. Then, an higher order method is used, namely, the Weighted Least Square (WLSQ) algorithm. This method is widely used in classical Navier-Stokes based immersed boundary wall modeling 5758 . The gradient is taken as the unknown, solved by minimizing the error function:

$$
J=\sum_{k}\left(w_{k} e_{k}\right)^{2}=\mathbf{e}^{T} \mathbf{W e},
$$

The weight $\mathbf{W}$ is the inverse distance to the boundary point, in order to give more influence to close points. The error minimization leads to:

$$
(\nabla \phi)_{B}=\left(\mathbf{D}^{T} \mathbf{W D}\right)^{-1} \mathbf{D}^{T} \mathbf{W}\left(\phi-\phi_{B}\right),
$$

where $\left(\mathbf{D}^{T} \mathbf{W D}\right)^{-1}$ is a $3 \times 3$ matrix for $3 \mathrm{D}$. 


\section{RESULTS}

In this section, the proposed HRR- $p$ model coupled with proper boundary conditions, a shock capturing technique and a turbulence model is assessed on different configurations and compared to reference solutions that can be either analytical solutions or previous numerical solutions obtained by validated flow solvers. The validations are aimed at demonstrating the ability of the present model to accurately reproduce fully compressible effects for a wide range of physical parameters. Unless it is specified, all the simulations are carried out with $\sigma=0.9$ and inviscid cases with a dynamic viscosity of $\mu=10^{-15}$. The classical definition for the acoustic Courant-Friedrichs-Lewy (CFL) number with respect to infinite state is used in this study:

$$
\mathrm{CFL}=\frac{\left|u_{\infty}\right|+c}{\Delta x / \Delta t}
$$

with the physical sound speed $c=\sqrt{\gamma r T_{\infty}}$. A necessary condition for stability is $\mathrm{CFL} \leq 1$. For each test case, this free parameter, to be tuned through a reference sound speed changing the time step $\Delta t$, will be precised.

Note that additional academic validation test cases are available in our initial publication ${ }^{9}$ and in a recent comparison between pressure-based and density-based solvers 59 , including in particular:

- Convection of Kovásznay modes (vortex, entropy, acoustic) up to Mach 4 for the first two,

- Couette flows up to Mach 3.3,

- Shock tubes problems,

- Shock/vortex interaction.

\section{A. Inviscid transonic flow over a $10 \%$ bump}

The first test case chosen to validate our model on a two-dimensional configuration involving non-straight walls and a Mach number close to 1 is a transonic flow over a $10 \%$ circular bump. The length of the domain is $L=3 \mathrm{~m}$ for a height of the channel $H=1 \mathrm{~m}$ on a $300 \times 100$ mesh. The flow at the inlet is injected in the $x$-direction corresponding to 
a Mach number $M=0.675$ with a total pressure of $P_{t}=10^{5} \mathrm{~Pa}$ and a total temperature $T_{t}=293.15 \mathrm{~K}$. At the outlet, NSCBC with a prescribed pressure, corresponding to the inlet conditions, is used to avoid spurious wave reflection into the domain 26. The other boundaries (top and bottom surfaces) are frictionless adiabatic walls. Simulation is performed with the condition $\mathrm{CFL}=0.4$. To properly capture the shock induced by the curved profile, a shock sensor is applied with $s_{c}=10$. Figure 1 shows the adimensional macroscopic quantities (density, pressure and Mach number) fields. A quantitative comparison is given in Fig. 2 where the Mach number on the horizontal lower wall is compared to a LU-SGS scheme using a AUSM+up flux ${ }^{60}$. It can be seen through these figures that the shock waves are properly captured by the method with few oscillations.
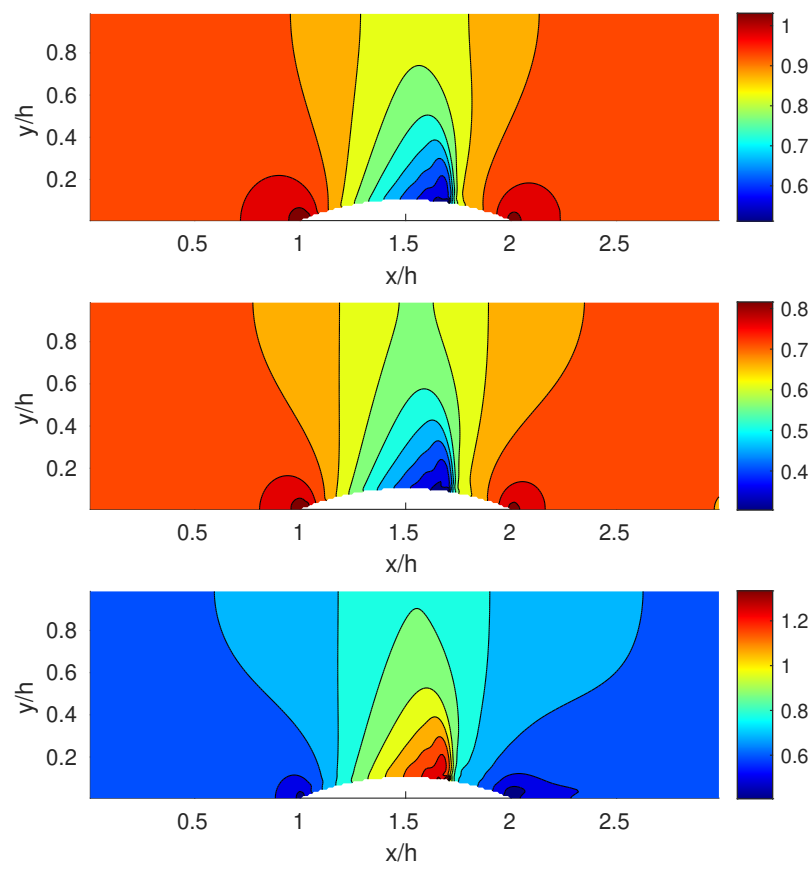

Figure 1: Density, Pressure and Mach number (respectively from top to bottom) fields. Axes are normalized using the height of the channel $h$.

\section{B. Inviscid supersonic flow over a $4 \%$ bump}

The second test case reproduces the same channel dimensions including a $4 \%$ circular bump with a $600 \times 200$ mesh grid. This configuration is showed here to assess the capability of the method to deal with supersonic flow including shock waves reflection 6162 . As for the previous test case, the bump is set in the middle of the channel. At the inlet, a supersonic 


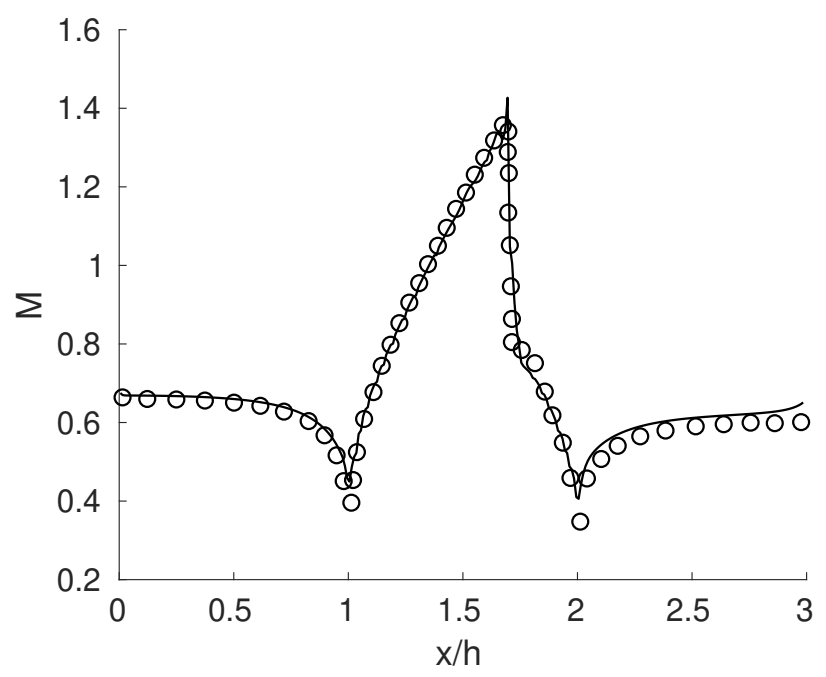

Figure 2: Mach number distribution along the lower wall. ○ LU-SGS, AUSM + up ; LBM results. $x$ axis is normalized using the height of the channel $h$.

condition is imposed with a pressure $p=101325 \mathrm{~Pa}$, a density $\rho=1 \mathrm{~kg} \mathrm{~m}^{-3}$ and a velocity parallel to the $x$-direction corresponding to a Mach number $M=1.4$. The CFL condition is $\mathrm{CFL}=0.4$ and the shock sensor coefficient is $s_{c}=3$. The top and bottom walls are set as free-slip adiabatic wall and due to the supersonic configuration, the outlet corresponds to a Neumann first order condition for each macroscopic variable.

Figure 3 shows the density, pressure and Mach number fields. It can be seen that the shock waves and reflections through the frictionless walls are properly captured without non-physical oscillations, exhibiting similar properties with previous work ${ }^{28}$. On Fig. 4 . values of Mach number are plotted along the top and bottom walls. These distributions are compared with reference simulation on a $90 \times 30$ nonuniform body-fitted grid, using Artificial Compression Method (ACM) with a second-order accuracy scheme ${ }^{63}$. Our results are in good agreement with this reference solution. Finally, a comparison is made concerning Mach number and pressure distributions along horizontal and vertical middle lines with a reference Jameson Schmidt Turkel second-order accurate finite volume scheme ${ }^{53}$. In this case, both solutions are computed with the same mesh size ${ }^{28}$. 

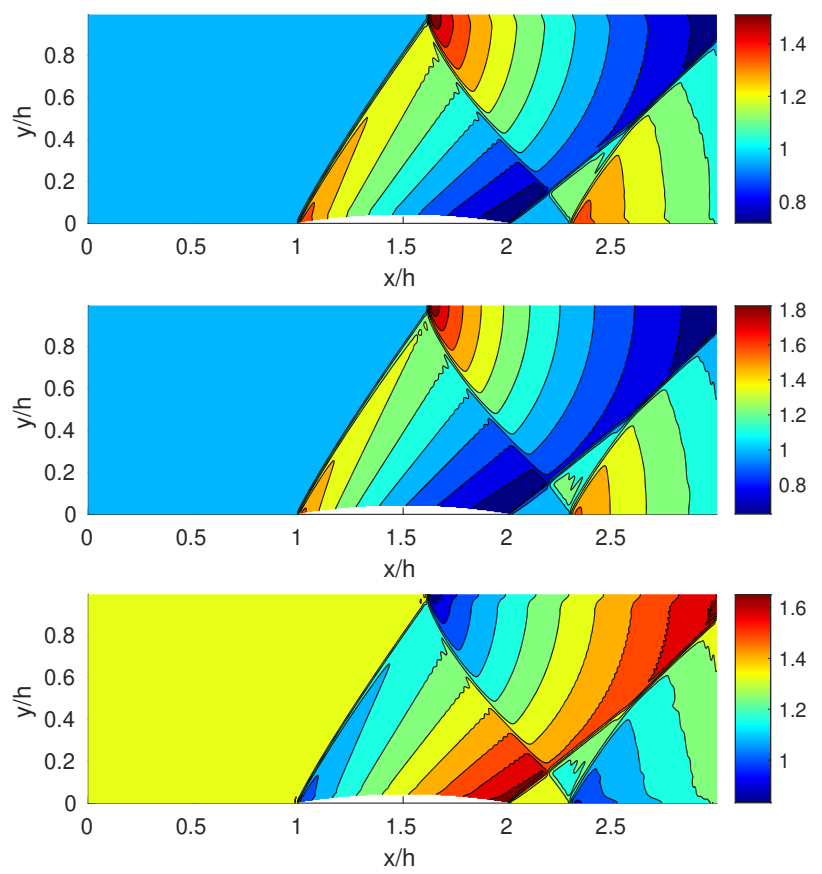

Figure 3: Density (top), Pressure (middle) and Mach number (bottom) fields. Axes are normalized using the height of the channel $h$.

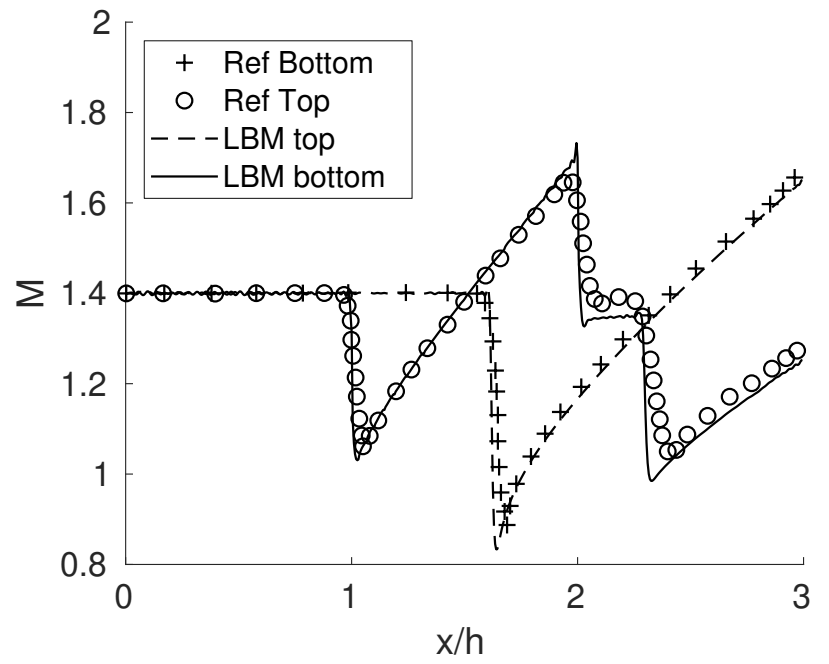

Figure 4: Mach number distribution along the top and lower walls. $x$ axis is normalized using the height of the channel $h$. 

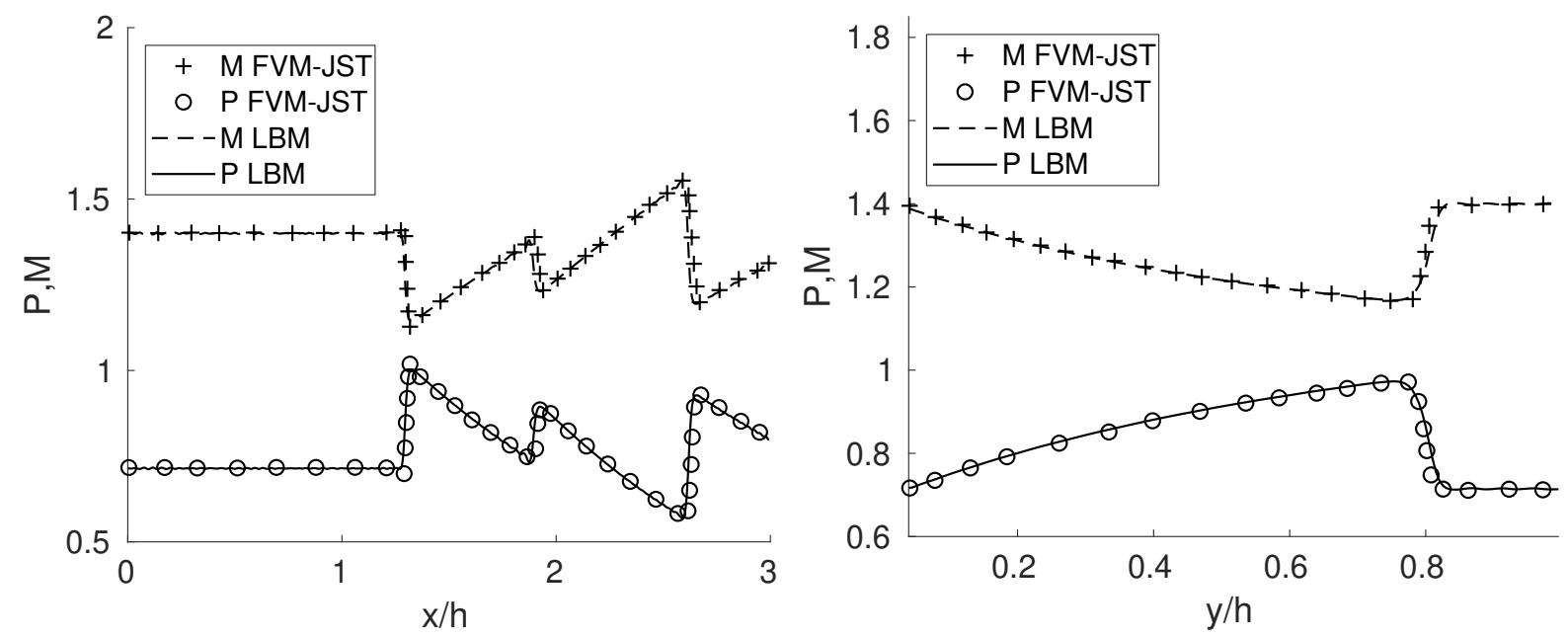

Figure 5: Mach number and pressure distributions along horizontal (left) and vertical

(right) mid-lines. Abscissa axes are normalized using the height of the channel $h$.

\section{Steady inviscid flow over a NACA0012 airfoil at Mach numbers between 0.6 and 1.2}

To increase the complexity of the configurations tested, a simulation of an inviscid flow over a NACA0012 airfoil is performed to deal with more complex geometrical boundaries including shock waves ${ }^{6465}$. The simulation domain is $x \in[-12,22] \times y \in[-12,12]$ on a $2720 \times 1920$ mesh including two refinement boxes to capture accurately the shock in the vicinity of the wing (320 nodes on the chord). The left and bottom boundaries correspond to subsonic inlets to give an angle of attack to the wing corresponding to $\alpha=1.25^{\circ}$ leading to $u_{\infty}=U_{0} \cos \alpha$ and $v_{\infty}=U_{0} \sin \alpha$ with $U_{0}$ calculated in agreement with the desired Mach number. Five configurations are tested with $M=0.6, M=0.75, M=0.8, M=0.9$ and $M=1.2$ at $\mathrm{CFL}=0.4$ to see the progression of shock waves increasing the Mach number.

The right and top boundaries correspond to outlet conditions, meaning characteristic conditions for the subsonic cases and a supersonic outlet for the $M=1.2$ case. Free-slip adiabatic condition is applied to the surface of the wing. All the open boundaries are coupled with sponge layers, relaxing all macroscopic variables toward a prescribed infinite state. The Mach number field is plotted on Fig. 6 for the different configurations along with the mesh refinement regions. A quantitative comparison is given on Fig. 7 using pressure coefficient, 
a dimensionless number describing the relative pressure on a surface:

$$
c_{p}=\frac{p-p_{\infty}}{\frac{1}{2} \rho_{\infty} \mathbf{u}_{\infty}^{2}}
$$

Our pressure coefficients are plotted together with a reference body fitted Euler solver solution 66 . The results of our algorithm are in good agreement with this reference, demonstrating the capability of the method to handle shock in a transonic regime for a complex $2 \mathrm{D}$ test case.

\section{Steady inviscid transonic flow over an ONERA M6 wing}

The complete model is now validated on a fully three dimensional test case with an ONERA M6 wing. It is a swept, semi-span wing with no twist. The free-stream Mach number is $M_{\infty}=0.8395$ including an angle of attack $\alpha=3.06^{\circ}$. This angle is given to the fluid at the inlet. The simulation domain is $x \in\left[-1.2 \times 10^{1} \mathrm{~m}, 2.2 \times 10^{1} \mathrm{~m}\right] \times y \in\left[0 . \mathrm{m}, 1.2 \times 10^{1} \mathrm{~m}\right] \times z \in$ $[-5.0 \mathrm{~m}, 5.0 \mathrm{~m}]$ on a $340 \times 120 \times 100$ mesh grid, including six rectangular mesh refinement boxes around the airfoil to simulate the flow around accurately in the interest region while keeping reasonable computation costs. Table. I introduces the setup and the exact geometry of mesh refinement areas. A visual description of the refinement setup is presented on Fig. 8

Table I: Setup and levels of refinement for ONERA M6 wing calculation: origin of the leading edge is located at $(0.0,0.0,0.0)$ and mean aerodynamic chord is $0.64607 \mathrm{~m}$

\begin{tabular}{||cccc||}
\hline \hline Level & Start position & End position & $\Delta x(\mathrm{~m})$ \\
\hline \hline 0 & $(-12.0,0.0,-5.0)$ & $(22.0,12.0,5.0)$ & 0.1 \\
\hline 1 & $(-3.0,0.0,-1.0)$ & $(5.0,5.0,1.0)$ & 0.05 \\
\hline 2 & $(-2.0,0.0,-0.5)$ & $(3.0,3.0,0.5)$ & 0.025 \\
\hline 3 & $(-1.0,0.0,-0.2)$ & $(2.0,2.0,0.2)$ & 0.0125 \\
\hline 4 & $(-0.3,0.0,-0.1)$ & $(1.5,1.5,0.1)$ & 0.00625 \\
\hline 5 & $(-0.1,0.0,-0.07)$ & $(1.3,1.3,0.07)$ & 0.003125 \\
\hline 6 & $(-0.05,0.0,-0.05)$ & $(1.25,1.25,0.05)$ & 0.0015625 \\
\hline \hline
\end{tabular}



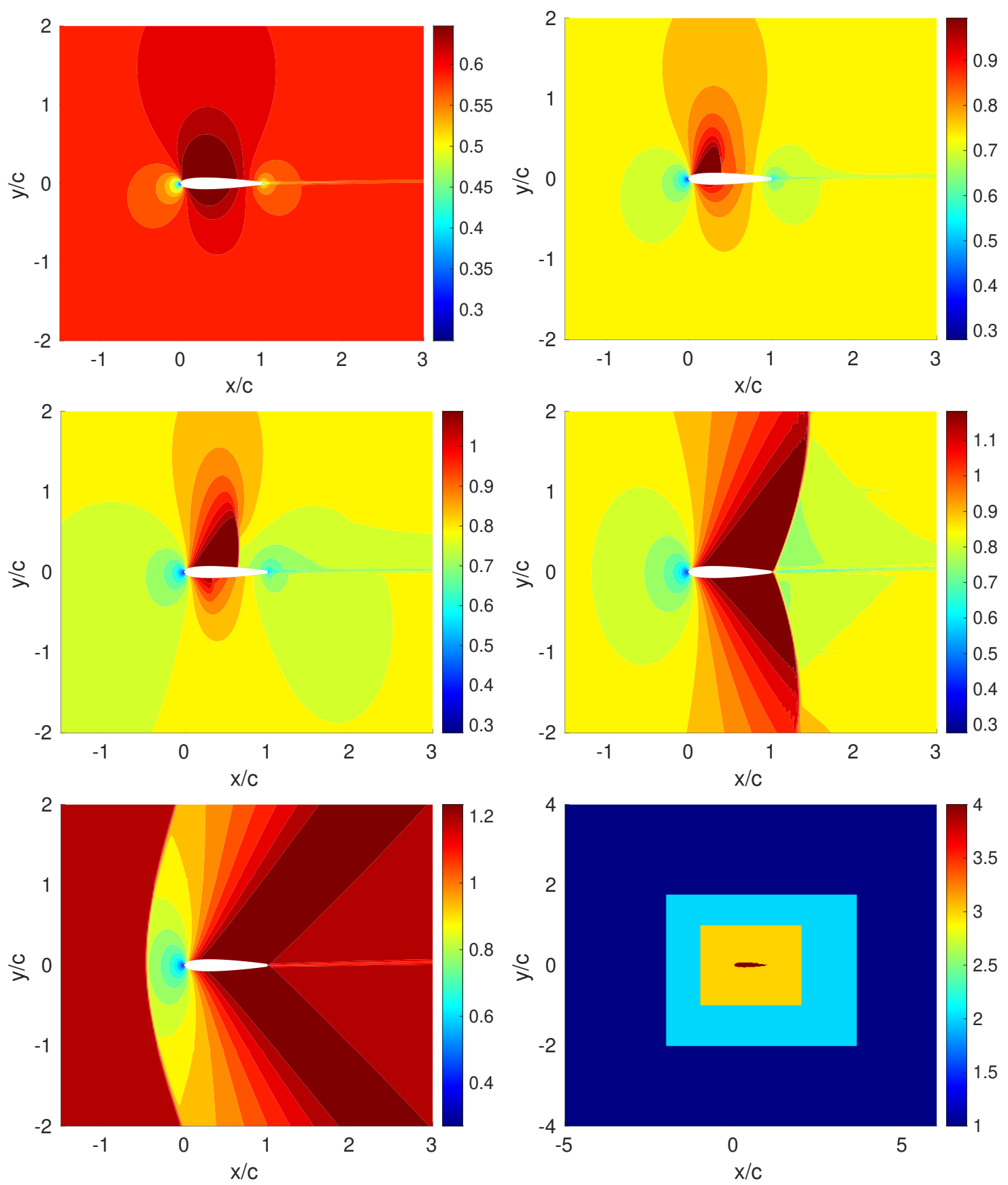

Figure 6: Mach number field for (from top-left to bottom-right): $M_{\infty}=0.6, M_{\infty}=0.75$, $M_{\infty}=0.8, M_{\infty}=0.9, M_{\infty}=1.2$. Axes are normalized using the chord of the wing $c$. The last plot indicates the mesh refinement regions around the wing: a growth on the colorbar represents a finer mesh and the maximum value indicates the solid body.

Free-stream macroscopic state is given at the inlet and bottom boundaries while a subsonic outlet with a prescribed pressure $p_{\infty}$ is imposed for the outflow to mimic a far field 


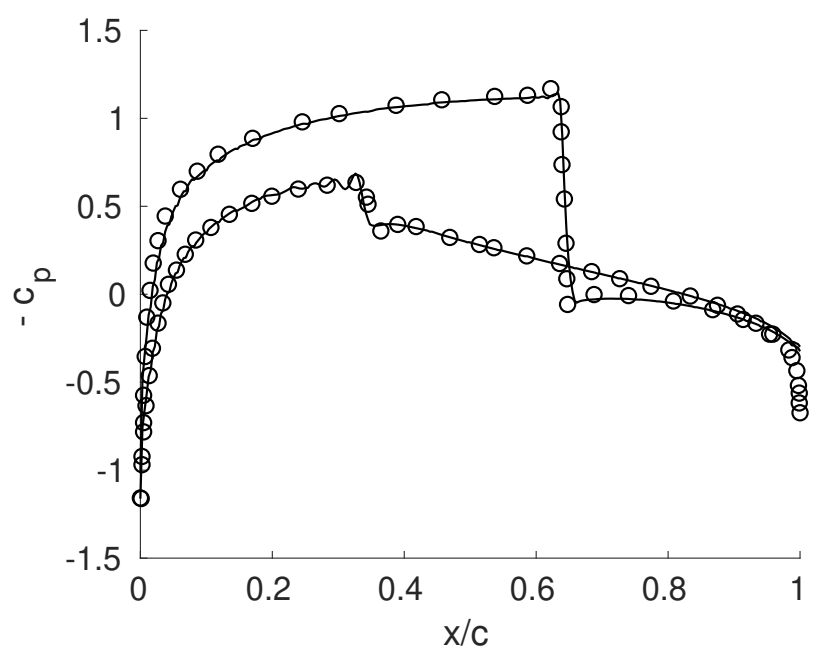

Figure 7: Pressure coefficient $c_{p}$ for $M_{\infty}=0.8$; - LBM results; ${ }^{666}$; $x$ axis is normalized using the chord of the wing $c$.

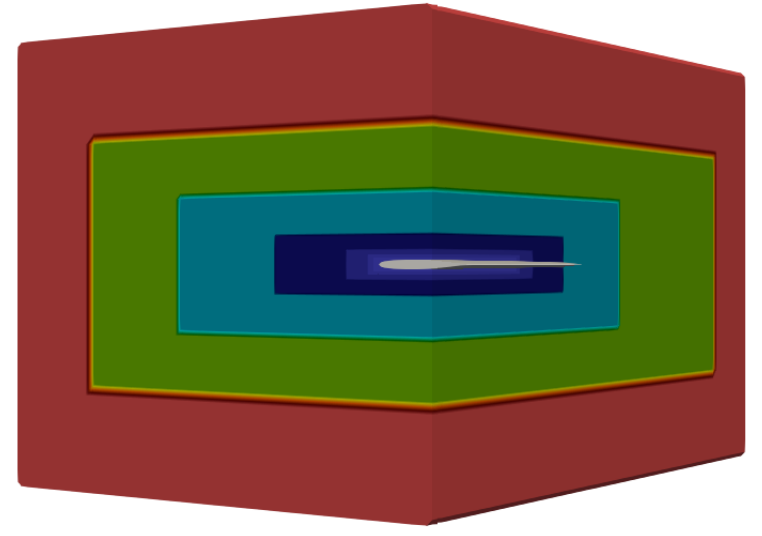

Figure 8: Mesh refinement regions with the smallest grid size around the wing and the largest one in the far field space.

condition. The airfoil and the wall attached to it both correspond to free-slip and adiabatic walls. Sponge zones with a relaxation on density $\rho$, temperature $T$ and velocity $\mathbf{u}$ are prescribed at inlet and outlet boundaries to kill the remaining spurious waves. Our simulation is compared, in terms of pressure coefficients, to a DG method of the literature ${ }^{67}$ on Fig. 9 This validates the method for 3D complex geometry in the scope of transonic regime. 

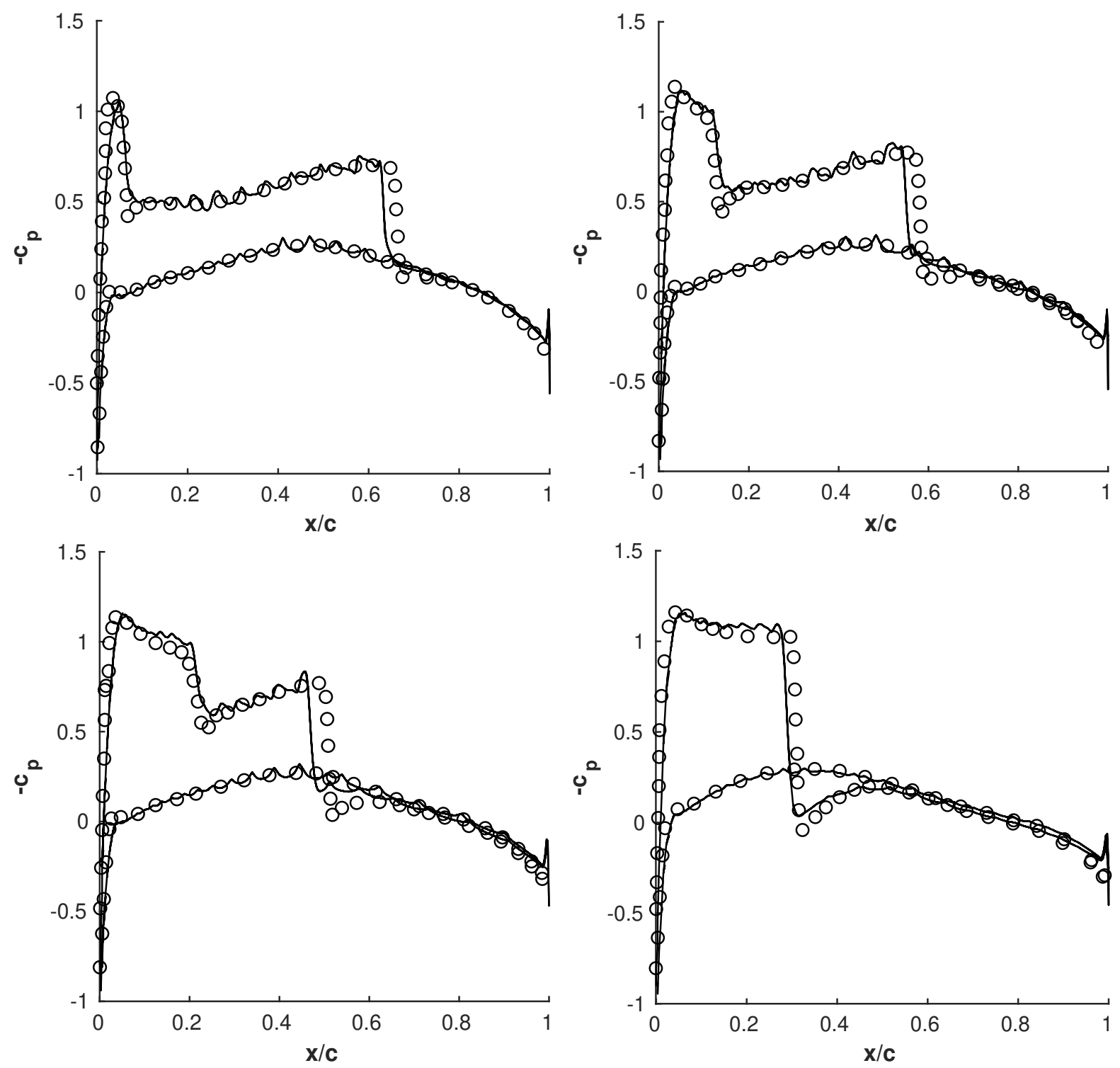

Figure 9: Pressure coefficient at (from top-left to bottom-right) $y=20 \%, y=44 \%$, $y=65 \%$ and $y=90 \%$ of the wing span; - LBM results; $\circ$ Euler Ref. $x$ axis is normalized using the chord of the wing $c$ at the spanwise considered section.

\section{E. Turbulent transonic flow over a ONERA M6 wing}

As final validation test case, we repeat the inviscid M6 wing configuration activating the Vremann turbulence model as well as the wall-law algorithm. The numerical parameters remain unchanged. The mesh was modified for the turbulent test case, adding one refinement level compared to the Euler configuration, to satisfy the wall-law $y^{+}$requirements (averaging at 200). Mesh is automatically generated, with the refinement boxes obtained by extrusion 
of the wing geometry (see Fig. 10). The resulting mesh consists of $6.3 \times 10^{7}$ elements, with $\delta x$ ranging from $7.8 \times 10^{-4} \mathrm{~m}$ at the wall to $0.1 \mathrm{~m}$ in the far field.

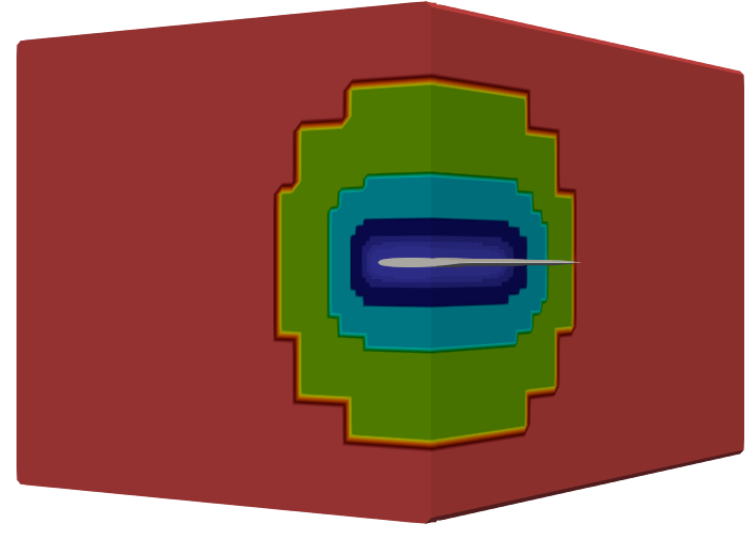

Figure 10: Mesh refinement regions with the smallest grid size around the wing and the largest one in the far field space.

The goal is to validate our shock capturing technique previously applied in combination with a LES turbulence model and a wall model68. This test case was designed by the ONERA Aerodynamics Departement, in 1972, as a reference to study three dimensional high Reynolds number flows. This became a traditional benchmark to assess the capability of a numerical method to deal with complex phenomena 69 .

The free-stream Mach number is $M_{\infty}=0.8395$ at $\mathrm{CFL}=0.4$ and the angle of attack $\alpha=3.06^{\circ}$. Viscosity is introduced through a Reynolds number $\operatorname{Re}=11.72 \times 10^{6}$ related to the mean aerodynamic chord $c=0.64607 \mathrm{~m}$. This configuration induces a typical lambda shock which should be well captured by the method.

The same inlet and outlet characteristic boundary conditions as for the euler M6 test case are used to give the proper angle to the flow at the inlet and to avoid spurious wave reflection at the outlet, prescribing an infinite pressure $p_{\infty}$. Sponge layers are also used. A Vreman subgrid model to calculate a turbulent dynamic viscosity and an explicit wall law are applied in order to properly capture the turbulence in the flow, separating small and large scales.

The pressure coefficient field on the surface of the wing is plotted on Fig. 11 where the topology of the lambda shock can be observed. This analysis is completed with a quantitative comparison of $c_{p}$ at different locations on the wing. During experiments, pressure orifices were present on the upper and lower surfaces of the wing, divided in seven sections as a 
function of the semi-span position. Our results are compared to the given data of this well-known experiment ${ }^{70}$ on Fig. 12 and are in good agreement with it.

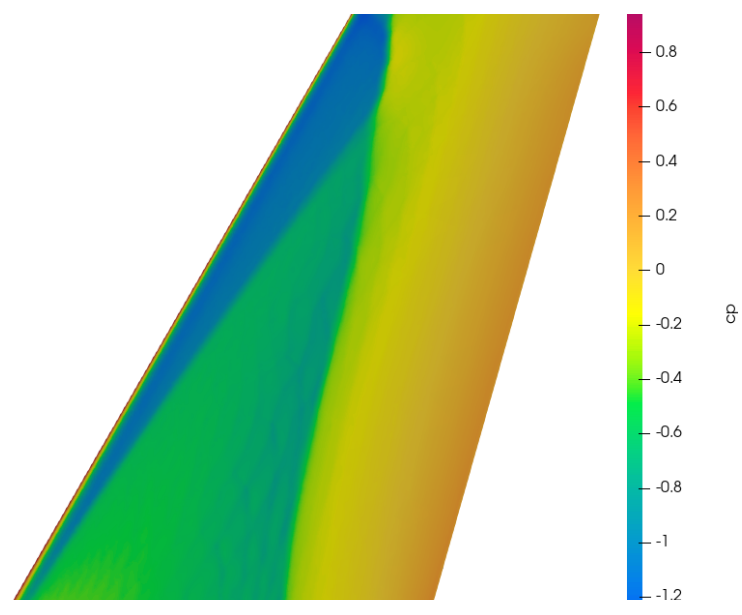

Figure 11: Pressure coefficient on the top surface of the ONERA M6 wing

\section{CONCLUDING REMARKS}

We have presented a number of tools and models compatible with our hybrid pressurebased Lattice-Boltzmann method ${ }^{9}$, significantly increasing its capabilities. To deal with complex aerodynamic geometries, an efficient treatment for wall and open boundaries has been coupled with a grid refinement technique. Large-Eddy Simulation (LES) has been introduced through a Vreman subgrid turbulence model and an improved law of the wall accounting for unresolved scales. The numerical setup also includes an artificial viscosity method to capture shocks at high Mach numbers and absorbing sponge terms to supplement NSCBC boundary conditions.

Equipped with these methods, the HRR-P LBM is assessed with a succession of increasingly complex test cases, ending with the first wall-law, large eddy Lattice-Boltzmann simulation of a full 3D transonic wing. These results allow to envision encouraging perspectives for both industrial and academic configurations. Future works include applications (i) to aero-acoustics, and (ii) to complex 3D supersonic flows.

When discontinuities are encountered at a Mach number higher than 1.5, only the HRRP LBM coupled with the total energy equation under a conservative format can lead to the correct Rankine Hugoniot jump relations. A first model including this feature has 

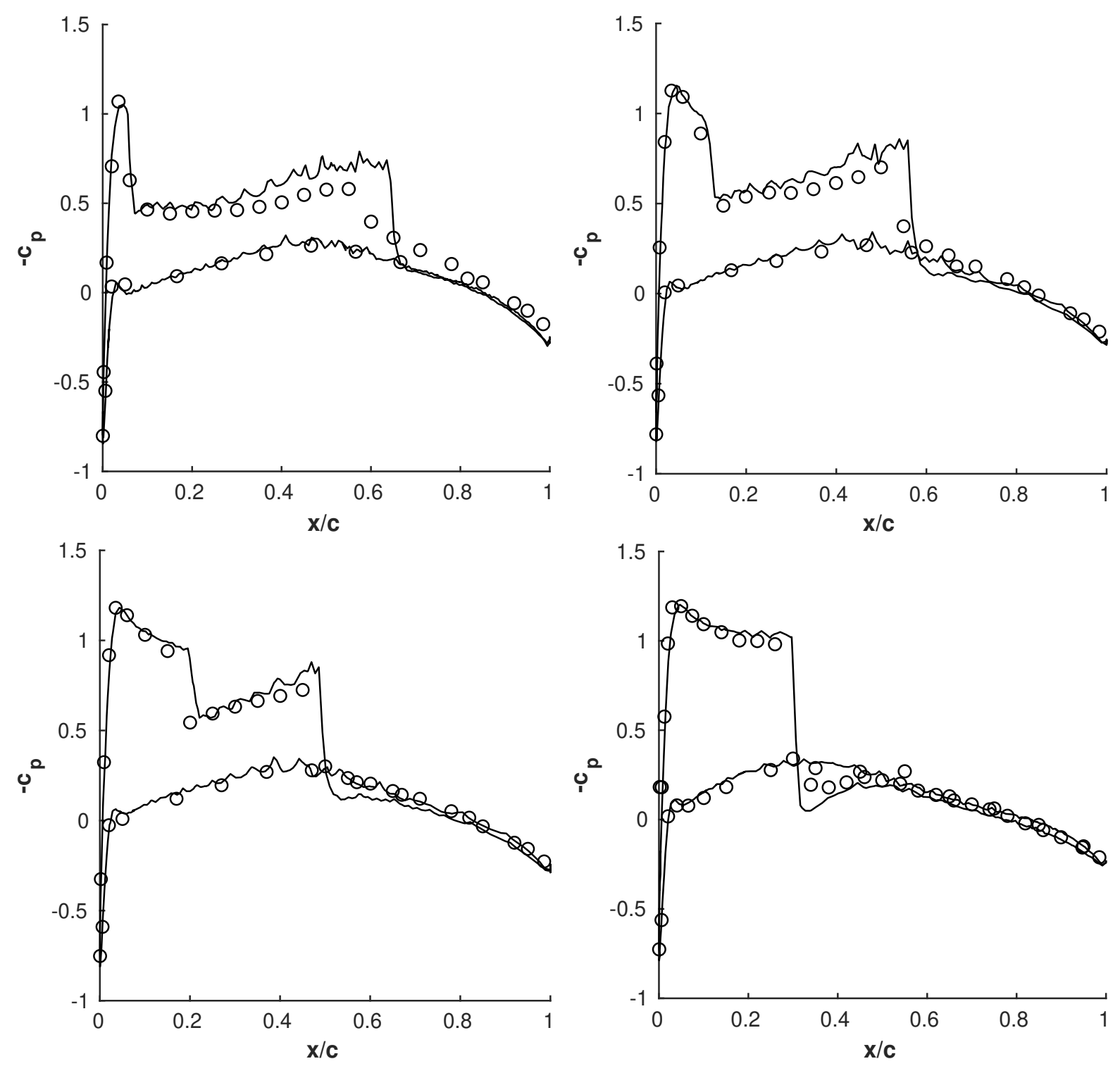

Figure 12: Pressure coefficient at (from top-left to bottom-right) $y=20 \%, y=44 \%$, $y=65 \%$ and $y=90 \%$ of the wing span; - LBM results; $\circ$ Expt. $x$ axis is normalized using the chord of the wing $c$ at the spanwise considered section.

been proposed and validated under academic configurations ${ }^{71}$ but the extension to complex applications including shocks at Mach numbers higher than 1.5 is still an open issue.

\section{ACKNOWLEDGEMENTS}

Part of this research was supported by ANR, Renault, Airbus and SafranTech by the Industrial Chair Program ALBUMS (ANR-CHIND-18-ALBUMS). Centre de Calcul 
Intensif d'Aix-Marseille and GENCI-TGCC/CINES (Grants 2021-A0092B11951, 2021A0092A07679) are acknowledged for granting access to their high performance computing resources.

\section{DATA AVAILABILITY}

The data that support the findings of this study are available from the corresponding author upon reasonable request.

\section{REFERENCES}

${ }^{1}$ S. Chen and G. D. Doolen, "Lattice boltzmann method for fluid flows," Annual review of fluid mechanics 30, 329-364 (1998).

2Z. Guo and C. Shu, Lattice Boltzmann method and its applications in engineering, Vol. 3 (World Scientific, 2013).

${ }^{3}$ T. Krüger, H. Kusumaatmaja, A. Kuzmin, O. Shardt, G. Silva, and E. M. Viggen, The Lattice Boltzmann Method: Principles and Practice (Springer, 2016).

${ }^{4} \mathrm{~S}$. Succi, The lattice Boltzmann equation: for complex states of flowing matter (Oxford University Press, 2018).

${ }^{5}$ Y. Feng, P. Boivin, J. Jacob, and P. Sagaut, "Hybrid recursive regularized lattice boltzmann simulation of humid air with application to meteorological flows," Physical Review E 100, 023304 (2019).

${ }^{6}$ C. G. Coreixas, High-order extension of the recursive regularized lattice Boltzmann method, Ph.D. thesis (2018).

${ }^{7}$ N. Frapolli, Entropic lattice Boltzmann models for thermal and compressible flows, Ph.D. thesis, ETH Zurich (2017).

${ }^{8}$ Y. Feng, P. Sagaut, and W.-Q. Tao, "A compressible lattice boltzmann finite volume model for high subsonic and transonic flows on regular lattices," Computers \& Fluids 131, 45-55 (2016).

${ }^{9}$ G. Farag, S. Zhao, T. Coratger, P. Boivin, G. Chiavassa, and P. Sagaut, "A pressure-based regularized lattice-boltzmann method for the simulation of compressible flows," Physics of Fluids 32, 066106 (2020). 
${ }^{10}$ S. Hosseini, A. Abdelsamie, N. Darabiha, and D. Thévenin, "Low-mach hybrid lattice boltzmann-finite difference solver for combustion in complex flows," Physics of Fluids 32, 077105 (2020).

${ }^{11}$ S. A. Hosseini, A. Eshghinejadfard, N. Darabiha, and D. Thévenin, "Weakly compressible lattice boltzmann simulations of reacting flows with detailed thermo-chemical models," Computers \& Mathematics with Applications 79, 141-158 (2020).

${ }^{12}$ M. Tayyab, S. Zhao, Y. Feng, and P. Boivin, "Hybrid regularized lattice-boltzmann modelling of premixed and non-premixed combustion processes," Combustion and Flame 211, 173-184 (2020).

${ }^{13}$ M. Tayyab, S. Zhao, and P. Boivin, "Lattice-boltzmann modelling of a turbulent bluffbody stabilized flame," Physics of Fluids 33, 031701 (2021).

${ }^{14} \mathrm{P}$. Boivin, M. Tayyab, and S. Zhao, "Benchmarking a lattice-boltzmann solver for reactive flows: Is the method worth the effort for combustion?" Physics of Fluids 33, 017703 (2021).

${ }^{15}$ L. Merlier, J. Jacob, and P. Sagaut, "Lattice-boltzmann large-eddy simulation of pollutant dispersion in street canyons including tree planting effects," Atmospheric Environment 195, 89-103 (2018).

${ }^{16}$ L. Merlier, J. Jacob, and P. Sagaut, "Lattice-boltzmann large-eddy simulation of pollutant dispersion in complex urban environment with dense gas effect: Model evaluation and flow analysis," Building and Environment 148, 634-652 (2019).

${ }^{17}$ I. Cheylan, S. Zhao, P. Boivin, and P. Sagaut, "Compressible pressure-based latticeboltzmann applied to humid air with phase change," Applied Thermal Engineering , 116868 (2021).

${ }^{18}$ Y. Feng, P. Boivin, J. Jacob, and P. Sagaut, "Hybrid recursive regularized thermal lattice boltzmann model for high subsonic compressible flows," Journal of Computational Physics 394, 82 - 99 (2019).

${ }^{19}$ G. Farag, T. Coratger, G. Wissocq, S. Zhao, P. Boivin, and P. Sagaut, "A unified hybrid lattice-boltzmann method for compressible flows: bridging between pressure-based and density-based methods," Physics of Fluids 33 (2021).

${ }^{20}$ D. Casalino, A. F. Ribeiro, E. Fares, and S. Nölting, "Lattice-boltzmann aeroacoustic analysis of the lagoon landing-gear configuration," AIAA journal 52, 1232-1248 (2014).

${ }^{21}$ A. Sengissen, J.-C. Giret, C. Coreixas, and J.-F. Boussuge, "Simulations of lagoon landinggear noise using lattice boltzmann solver," in 21st AIAA/CEAS Aeroacoustics Conference 
(2015) p. 2993.

${ }^{22}$ N. I. Prasianakis and I. V. Karlin, "Lattice boltzmann method for thermal flow simulation on standard lattices," Physical Review E 76, 016702 (2007).

${ }^{23}$ C.-H. Liu, K.-H. Lin, H.-C. Mai, and C.-A. Lin, "Thermal boundary conditions for thermal lattice boltzmann simulations," Computers \& Mathematics with Applications 59, 2178 $2193(2010)$.

${ }^{24}$ N. Frapolli, S. Chikatamarla, and I. Karlin, "Multispeed entropic lattice boltzmann model for thermal flows," Physical Review E 90, 043306 (2014).

${ }^{25}$ G. Di Ilio, D. Chiappini, S. Ubertini, G. Bella, and S. Succi, "Fluid flow around naca 0012 airfoil at low-reynolds numbers with hybrid lattice boltzmann method," Computers \& Fluids 166, 200-208 (2018).

${ }^{26}$ Y. Feng, S. Guo, J. Jacob, and P. Sagaut, "Solid wall and open boundary conditions in hybrid recursive regularized lattice boltzmann method for compressible flows," Physics of Fluids 31, 126103 (2019).

${ }^{27}$ J. Latt, C. Coreixas, J. Beny, and A. Parmigiani, "Efficient supersonic flow simulations using lattice boltzmann methods based on numerical equilibria," Philosophical Transactions of the Royal Society A 378, 20190559 (2020).

${ }^{28}$ S. Guo, Y. Feng, J. Jacob, F. Renard, and P. Sagaut, "An efficient lattice boltzmann method for compressible aerodynamics on d3q19 lattice," Journal of Computational Physics , 109570 (2020).

${ }^{29} \mathrm{~K}$. Hejranfar and A. Ghaffarian, "A high-order accurate unstructured spectral difference lattice boltzmann method for computing inviscid and viscous compressible flows," Aerospace Science and Technology 98, 105661 (2020).

${ }^{30}$ M. H. Saadat, F. Bösch, and I. V. Karlin, "Lattice boltzmann model for compressible flows on standard lattices: Variable prandtl number and adiabatic exponent," Physical Review E 99, 013306 (2019).

${ }^{31}$ M. H. Saadat, B. Dorschner, and I. Karlin, "Extended lattice boltzmann model," Entropy 23, 475 (2021).

${ }^{32}$ L. Yang, C. Shu, and J. Wu, "A hybrid lattice boltzmann flux solver for simulation of 3d compressible viscous flows," in eighth international conference on computational fluid dynamics, Chengdu, China (2014) pp. 14-18. 
${ }^{33}$ Y. Feng, S. Guo, J. Jacob, and P. Sagaut, "Grid refinement in the three-dimensional hybrid recursive regularized lattice boltzmann method for compressible aerodynamics," Physical Review E 101, 063302 (2020).

${ }^{34}$ O. Filippova and D. Hänel, "Grid refinement for lattice-bgk models," Journal of Computational physics 147, 219-228 (1998).

${ }^{35}$ D. Lagrava, O. Malaspinas, J. Latt, and B. Chopard, "Advances in multi-domain lattice boltzmann grid refinement," Journal of Computational Physics 231, 4808-4822 (2012).

${ }^{36}$ J. Jacob, O. Malaspinas, and P. Sagaut, "A new hybrid recursive regularised bhatnagargross-krook collision model for lattice boltzmann method-based large eddy simulation," Journal of Turbulence , 1-26 (2018).

${ }^{37}$ J. Degrigny, S.-G. Cai, J.-F. Boussuge, and P. Sagaut, "Improved wall model treatment for aerodynamic flows in lbm," Computers \& Fluids , 105041 (2021).

${ }^{38}$ S.-G. Cai, J. Degrigny, J.-F. Boussuge, and P. Sagaut, "Coupling of turbulence wall models and immersed boundaries on cartesian grids," Journal of Computational Physics 429, 109995 (2021).

${ }^{39}$ P. L. Bhatnagar, E. P. Gross, and M. Krook, "A model for collision processes in gases. i. small amplitude processes in charged and neutral one-component systems," Phys. Rev. 94, 511-525 (1954).

${ }^{40}$ G. Farag, S. Zhao, G. Chiavassa, and P. Boivin, "Consistency study of lattice-boltzmann schemes macroscopic limit," Physics of Fluids 33, 037101 (2021).

${ }^{41}$ Y. Feng, P. Sagaut, and W. Tao, "A three dimensional lattice model for thermal compressible flow on standard lattices," Journal of Computational Physics 303, 514-529 (2015).

${ }^{42}$ J. Latt and B. Chopard, "Lattice boltzmann method with regularized pre-collision distribution functions," Mathematics and Computers in Simulation 72, 165-168 (2006).

${ }^{43}$ O. Malaspinas, "Increasing stability and accuracy of the lattice boltzmann scheme: recursivity and regularization," arXiv preprint arXiv:1505.06900 (2015).

${ }^{44}$ G. Wissocq and P. Sagaut, "Hydrodynamic limits and numerical errors of isothermal lattice boltzmann schemes," (2021), arXiv:2104.14217 [physics.flu-dyn].

${ }^{45}$ S. Zhao, G. Farag, P. Boivin, and P. Sagaut, "Toward fully conservative hybrid lattice boltzmann methods for compressible flows," Physics of Fluids 32, 126118 (2020).

${ }^{46} \mathrm{P}$. Sagaut, Large eddy simulation for incompressible flows: an introduction (Springer Science \& Business Media, 2006). 
${ }^{47}$ B. Vreman, B. Geurts, and H. Kuerten, "Large-eddy simulation of the turbulent mixing layer," Journal of fluid mechanics 339, 357-390 (1997).

${ }^{48}$ A. Vreman, "An eddy-viscosity subgrid-scale model for turbulent shear flow: Algebraic theory and applications," Physics of fluids 16, 3670-3681 (2004).

${ }^{49}$ E. Garnier, N. Adams, and P. Sagaut, Large eddy simulation for compressible flows (Springer Science \& Business Media, 2009).

${ }^{50}$ S.-G. Cai and P. Sagaut, "Explicit wall models for large eddy simulation," Physics of Fluids 33, 041703 (2021).

${ }^{51}$ S. R. Allmaras and F. T. Johnson, "Modifications and clarifications for the implementation of the spalart-allmaras turbulence model," in Seventh international conference on computational fluid dynamics (ICCFD'7) (Big Island, HI, 2012) pp. 1-11.

${ }^{52}$ E. Hewitt and R. E. Hewitt, "The gibbs-wilbraham phenomenon: an episode in fourier analysis," Archive for history of Exact Sciences 21, 129-160 (1979).

${ }^{53}$ A. Jameson, "Origins and further development of the jameson-schmidt-turkel scheme," AIAA Journal 55, 1487-1510 (2017).

${ }^{54}$ T. J. Poinsot and S. Lelef, "Boundary conditions for direct simulations of compressible viscous flows," Journal of computational physics 101, 104-129 (1992).

${ }^{55} \mathrm{H}$. Xu and P. Sagaut, "Analysis of the absorbing layers for the weakly-compressible lattice boltzmann methods," Journal of Computational Physics 245, 14-42 (2013).

${ }^{56}$ S. Wilhelm, J. Jacob, and P. Sagaut, "An explicit power-law-based wall model for lattice boltzmann method-reynolds-averaged numerical simulations of the flow around airfoils," Physics of Fluids 30, 065111 (2018).

${ }^{57}$ F. Capizzano, "Turbulent wall model for immersed boundary methods," AIAA journal 49, 2367-2381 (2011).

${ }^{58}$ Y. Tamaki, M. Harada, and T. Imamura, "Near-wall modification of spalart-allmaras turbulence model for immersed boundary method," AIAA journal 55, 3027-3039 (2017).

${ }^{59}$ G. Farag, T. Coratger, G. Wissocq, S. Zhao, P. Boivin, and P. Sagaut, "A unified hybrid lattice-boltzmann method for compressible flows: Bridging between pressure-based and density-based methods," Physics of Fluids 33, 086101 (2021).

${ }^{60}$ J. Fürst, "On the implicit density based openfoam solver for turbulent compressible flows," in EPJ Web of Conferences, Vol. 143 (EDP Sciences, 2017) p. 02027. 
${ }^{61}$ M. Allahyari, K. Yousefi, V. Esfahanian, and M. Darzi, "A block-interface approach for high-order finite-difference simulations of compressible flows," Journal of Applied Fluid Mechanics 14, 345-359 (2021).

${ }^{62} \mathrm{~A}$. Karbalaei and K. Hejranfar, "A central difference finite volume lattice boltzmann method for simulation of $2 \mathrm{~d}$ inviscid compressible flows on triangular meshes," in $A S M E$ International Mechanical Engineering Congress and Exposition, Vol. 52101 (American Society of Mechanical Engineers, 2018) p. V007T09A025.

${ }^{63}$ M. H. Djavareshkian and M. A. Jahdi, "Shock-capturing method using characteristic-based dissipation filters in pressure-based algorithm," Acta Mechanica 209, 99-113 (2010).

${ }^{64}$ N. Frapolli, S. S. Chikatamarla, and I. V. Karlin, "Entropic lattice boltzmann model for gas dynamics: Theory, boundary conditions, and implementation," Physical Review E 93, 063302 (2016).

${ }^{65}$ L. Ramírez, X. Nogueira, P. Ouro, F. Navarrina, S. Khelladi, and I. Colominas, "A higherorder chimera method for finite volume schemes," Archives of Computational Methods in Engineering 25, 691-706 (2018).

${ }^{66}$ V. G. Ferreira, R. A. de Queiroz, M. A. C. Candezano, G. A. Lima, L. Corrêa, C. M. Oishi, and F. L. Santos, "Simulation results and applications of an advection bounded scheme to practical flows," Computational \& Applied Mathematics 31, 591-616 (2012).

${ }^{67}$ J. J. van der Vegt and H. Van der Ven, "Discontinuous galerkin finite element method with anisotropic local grid refinement for inviscid compressible flows," Journal of Computational Physics 141, 46-77 (1998).

${ }^{68} \mathrm{M}$. Tayyab, S. Zhao, and P. Boivin, "Lattice-boltzmann modeling of a turbulent bluff-body stabilized flame," Physics of Fluids 33, 031701 (2021).

${ }^{69}$ N. Frapolli, S. Chikatamarla, and I. Karlin, "Theory, analysis, and applications of the entropic lattice boltzmann model for compressible flows," Entropy 22, 370 (2020).

${ }^{70} \mathrm{~V}$. Schmitt, "Pressure distributions on the onera m6-wing at transonic mach numbers, experimental data base for computer program assessment," AGARD AR-138 (1979).

${ }^{71}$ S. Zhao, G. Farag, P. Boivin, and P. Sagaut, "Toward fully conservative hybrid lattice boltzmann methods for compressible flows," Physics of Fluids 32, 126118 (2020).

${ }^{72}$ E. F. Toro, Riemann solvers and numerical methods for fluid dynamics: a practical introduction (Springer Science \& Business Media, 2009). 


\section{Appendix A: Forcing terms}

Depending on the order of the Gauss-Hermite quadrature ${ }^{3}$ used in the LB model, an adequate forcing term should be added to achieve a correct viscous stress tensor:

$$
a_{\alpha \beta}^{\mathrm{neq}} \approx-\Pi_{\alpha \beta}=-\mu\left(\frac{\partial u_{\alpha}}{\partial x_{\beta}}+\frac{\partial u_{\beta}}{\partial x_{\alpha}}-\frac{2}{D} \frac{\partial u_{\gamma}}{\partial x_{\gamma}} \delta_{\alpha \beta}\right)
$$

with $D$ the spatial dimension. For the $D 3 Q 19 r$ basis, the projected forcing term reads as

$$
a_{\alpha \beta}^{F^{E}}=c_{s}^{2} u_{\alpha}\left[\frac{\partial(\rho(1-\theta))}{\partial x_{\beta}}\right]+c_{s}^{2} u_{\beta}\left[\frac{\partial(\rho(1-\theta))}{\partial x_{\alpha}}\right]+\delta_{\alpha \beta} \rho c_{s}^{2} \frac{2}{D} \frac{\partial u_{\gamma}}{\partial x_{\gamma}}-a_{\alpha \beta}^{\text {cor }}+a_{\alpha \beta}^{F_{D}}
$$

where $a_{\alpha \beta}^{\text {cor }}$ is a correction tensor due to the deflection of second order moments of the population introduced by the modification of the mass equation, which can be evaluated as

$$
a_{\alpha \beta}^{\mathrm{cor}} \equiv c_{s}^{2} \delta_{\alpha \beta} \frac{\partial(\rho(1-\theta))}{\partial t}
$$

which can be discretized using a backward Euler operator and $a_{\alpha \beta}^{F_{D}}$ the correction tensor due to the defect of the lattice at third order

$$
a_{\alpha \beta}^{F_{D}}=-\left(\begin{array}{ccc}
\left(\rho u_{x}^{3}\right)_{, x} & \left(\rho u_{x} u_{y} u_{z}\right)_{, z} & \left(\rho u_{x} u_{y} u_{z}\right)_{, y} \\
\left(\rho u_{x} u_{y} u_{z}\right)_{, z} & \left(\rho u_{y}^{3}\right)_{, y} & \left(\rho u_{x} u_{y} u_{z}\right)_{, x} \\
\left(\rho u_{x} u_{y} u_{z}\right)_{, y} & \left(\rho u_{x} u_{y} u_{z}\right)_{, x} & \left(\rho u_{z}^{3}\right)_{, z}
\end{array}\right)
$$

where all the differential operations are performed using first order upwind FD except for the divergence operator for which a second order centered FD scheme was employed. The final expression of the forcing term is then

$$
F_{i}^{E}=\frac{\omega_{i}}{2 c_{s}^{4}} \mathcal{H}_{i, \alpha \beta}^{(2)} a_{\alpha \beta}^{F^{E}}
$$




\section{Appendix B: Recursive reconstruction of third order off-equilibrium tensor}

The third order non-equilibrium tensor is achieved via a recursive procedure from second order tensor, and reads as

$$
\begin{aligned}
& a_{\alpha \beta \gamma}^{(3) \text {,neq }}=u_{\alpha} \tilde{a}_{\beta \gamma}^{\text {neq }}+u_{\beta} \tilde{a}_{\alpha \gamma}^{\text {neq }}+u_{\gamma} \tilde{a}_{\alpha \beta}^{\text {neq }} \\
& a_{1}^{(3 r), \text { neq }} \equiv a_{x x y}^{(3), \text { neq }}+a_{y z z}^{(3), \text { neq }} \\
& a_{2}^{(3 r), \text { neq }} \equiv a_{x z z}^{(3) \text {,neq }}+a_{x y y}^{(3), \text { neq }} \\
& a_{3}^{(3 r), \text { neq }} \equiv a_{y y z}^{(3) \text {,neq }}+a_{x x z}^{(3) \text { neq }} \\
& a_{4}^{(3 r), \text { neq }} \equiv a_{x x y}^{(3) \text {,neq }}-a_{y z z}^{(3), \text { neq }} \\
& a_{5}^{(3 r), \text { neq }} \equiv a_{x z z}^{(3) \text {,neq }}-a_{x y y}^{(3), \text { neq }} \\
& a_{6}^{(3 r), \text { neq }} \equiv a_{y y z}^{(3), \text { neq }}-a_{x x z}^{(3), \text { neq }}
\end{aligned}
$$

\section{Appendix C: MUSCL-Hancock method}

Let's consider the following $1 \mathrm{D}$ transport equation

$$
\frac{s_{i}^{n+1}-s_{i}^{n}}{\Delta t}+\frac{F\left(s_{i+\frac{1}{2}}^{n}\right)-F\left(s_{i-\frac{1}{2}}^{n}\right)}{\Delta x}=0 .
$$

MUSCL-Hancock intercell fluxes $F\left(s_{i+\frac{1}{2}}^{n}\right)$ and $F\left(s_{i-\frac{1}{2}}^{n}\right)$ for a non-conservative form are computed as follows :

i) Data reconstruction. Evaluate the extrapolated intercell values at the left and right sides of the $i$ th cell as

$$
s_{i, L}=s_{i}-\frac{1}{2} \phi_{i} \Delta_{i}, \quad s_{i, R}=s_{i}+\frac{1}{2} \phi_{i} \Delta_{i}
$$

with $\Delta_{i}$ the local approximated slope

$$
\Delta_{i}=\frac{1}{2}\left[(1+\kappa)\left(s_{i}-s_{i-1}\right)+(1-\kappa)\left(s_{i+1}-s_{i}\right)\right]
$$

and $\phi_{i}$ a Van Albada flux limiter, only used for shocked flows, which reads

$$
\phi_{i}=\frac{r(r+1)}{1+r^{2}}, \quad r=\frac{s_{i}-s_{i-1}}{s_{i+1}-s_{i}} .
$$

In order to improve the accuracy, it was chosen to evaluate dynamically $\kappa=$ $\frac{1}{3}\left[\frac{2 \Delta t u_{i}}{\Delta x}-\operatorname{sign}\left(u_{i}\right)\right]$, leading to a third-order accurate scheme in both space and time for a constant $u_{i}$ value ${ }^{72}$. 
ii) Data evolution. Let the extrapolated boundary values evolve of a time $\frac{\Delta t}{2}$ according to

$$
\begin{aligned}
& \bar{s}_{i, L}=s_{i, L}+\frac{\Delta t u_{i}}{2 \Delta x}\left(s_{i, L}-s_{i, R}\right), \\
& \bar{s}_{i, R}=s_{i, R}+\frac{\Delta t u_{i}}{2 \Delta x}\left(s_{i, L}-s_{i, R}\right) .
\end{aligned}
$$

iii) Solution of the piece-wise constant problem. Finally we simply compute $F\left(s_{i+\frac{1}{2}}^{n}\right)$ and $F\left(s_{i-\frac{1}{2}}^{n}\right)$ using :

$$
\begin{aligned}
& F\left(s_{i+\frac{1}{2}}^{n}\right)= \begin{cases}u_{i} \bar{s}_{i, R} & \text { if } u_{i} \geq 0 \\
u_{i} \bar{s}_{i+1, L} & \text { if } u_{i}<0\end{cases} \\
& F\left(s_{i-\frac{1}{2}}^{n}\right)= \begin{cases}u_{i} \bar{s}_{i-1, R} & \text { if } u_{i} \geq 0 \\
u_{i} \bar{s}_{i, L} & \text { if } u_{i}<0\end{cases}
\end{aligned}
$$

Note that this scheme uses a 5-points stencil because the evaluation of the slope is non-local. 